\title{
Damping in a row of locally-resonant inclusions: dynamic homogenization and scattering of transient shear waves
}

\author{
Marie Touboul $^{\mathrm{a}}$, Xinzhao Gao ${ }^{\mathrm{b}}$, Bruno Lombard ${ }^{\mathrm{a}}$ \\ ${ }^{a}$ Aix Marseille Univ, CNRS, Centrale Marseille, LMA UMR 7031, Marseille, France \\ ${ }^{b}$ Southwest Jiaotong University, Chengdu, Sichuan, China
}

\begin{abstract}
The scattering of scalar waves by a periodic row of inclusions is theoretically and numerically investigated. The wavelength in the background medium is assumed to be much larger than the typical sizes of the inclusions. The latter are also much softer than the matrix, yielding localized resonances within the microstructure. Previous works in the inviscid case have concerned: (i) the derivation of effective resonant jump conditions, that are non local in time (Touboul et al., J. Elasticity, 2020); (ii) the introduction of auxiliary fields along the interface, providing a time-domain formulation of the scattering problem (Touboul et al., J. Comput. Phys., 2020). The present contribution extends the analysis to dissipative cases, which allows to be closer from real devices. The effective jump conditions with damping are obtained, both in the frequency domain and in the time domain. An exact plane-wave solution is proposed. A balance of energy is written, and new auxiliary fields are introduced. Practical implementation of the simulation methods is discussed. Then, numerical experiments are proposed to validate the auxiliary-field approach. The effect of dissipation is examined, and the relevance of the homogenized simulations in comparison with full-field simulations of transient waves is assessed. As an application, a numerical experiment of Coherent Perfect Absorption is finally proposed: at critical values of the attenuation parameter and close to the resonant frequencies, the waves impacting the dissipative resonant interface are fully absorbed.
\end{abstract}

Keywords: resonant microstructures, dissipation, homogenization, matched-asymptotic expansions, time-domain numerical methods.

\section{Introduction}

The design of microstructured media allows to control wave propagation in a fine way and leads to exotic effects, e.g. negative refraction, subwavelength imaging, lensing and cloaking, to cite a few. It constitutes the paradigm of metamaterials, which have undergone spectacular developments since the early 2000: see [7] and references therein for an overview.

Interesting effects can be reached when local resonances occur within the microstructure. These resonances can be induced by particular geometries (e.g. split ring resonators, Helmholtz resonators) or by a large contrast of physical parameters [1,2]. In electromagnetism, they yield doubly negative metamaterials on a range of frequencies, paving the way to negative refraction (see chapter 1-4 in [7]). In elasticity, the resonances are exploited to maximise sound absorption $[25,37,11,29]$ or to mitigate waves [39].

Either in non-resonant or resonant cases, an active direction of research concerns the size reduction of microstructures. It is often advantageous to replace a volumic distribution by a surfacic (in 3D) or a 
lineic (in 2D) distribution of scatterers. This is particularly important in the design of noise reduction devices, including sound insulation panels.

With such applications in mind, it is important to examine the competition between the energy leakage of resonant scatterers and the intrinsic dissipation losses [12, 20]. Discrete systems and the mitigation effects of broadband waves at subwavelength scales are examined in [5, 30]. The two mechanisms of the energy absorption (resonance of the inclusions yielding band-gaps, and dissipative effects in the materials) are discussed in [16, 19]. The Coherent Perfect Absorption of dissipative resonant metasurfaces (especially based on Helmholtz resonators) has motivated series of papers[35, 13, 14, 15, 17, 33, 34]. In all these works, the analysis of the scattering matrix is based on the calculation of the wave field at the microstructure scale, which can be computationnaly cumbersome.

An approach based on homogenisation may therefore be an interesting alternative. The twoscale homogenization method is well-known to simulate wave propagation in volumic microstructured media [3, 36]. It avoids having to mesh fine spatial scales and thus leads to enormous computational gains compared with brute-force simulations. However, the usual homogenization methods fail when considering a thin row of scatterers, because of the fast-scale fields close to the microstructure. To recover their efficiency, these methods must then be combined with matched-asymptotic expansions [26], yielding effective jump conditions on an equivalent meta-interface.

In elasticity, works on interface homogenization focused first on rows of non-resonant inclusions $[9,28,8,27]$, and then on resonant inclusions [31, 29, 41]. In [31, 41], two-scale asymptotic method has been combined with matched-asymptotic expansions to yield effective jump conditions, both in the frequency domain [31] and in the time domain [41]; in the latter case, the jump conditions turn out to be non-local. Second-order accuracy in terms of the small ratio $k_{m} h$ has been reached, where $k_{m}$ is the wavenumber in the background medium and $h$ is the typical size of the inclusions. The conservation of energy has been proven provided the effective interface is sufficiently enlarged. In [40], the formalism of auxiliary fields has been introduced to transform convolution operators at the interfaces into a set of local-in-time problems. This made it possible to build efficient numerical methods to simulate the scattering of transient shear waves by a row of resonant inclusions replaced by an effective interface. Comparisons between microstructured and homogenized fields have been successfully performed.

In the majority of these works on interface homogenisation, dissipation phenomena were neglected. A notable exception is given in [31]: the derivation of the interface conditions is done without attenuation, but damping is introduced heuristically in the numerical experiments (section 3 of this reference). We follow the line of this work, but with the additional objective of incorporating damping in the homogenisation process. Another objective is to propose time-domain formulations of the scattering phenomena.

Many energy dissipation mechanisms are involved in real experiments: damping due to the bonding between microstructure and the matrix, viscous boundary layers, intrinsic viscoelasticity, etc. The aim here is not to describe a particular mechanism in detail, but rather to examine the overall effect of energy dissipation on the resonance peaks of local-resonant inclusions. As is usual in structural dynamics, a phenomenological model is therefore considered. In this framework, the results presented in [41, 40] are modified and need to be re-examined on their main features: (i) effective jump conditions, (ii) energy balance, (iii) auxiliary fields, and (iv) discretization of the interfaces. These four points are addressed here. For the sake of brevity, the reader is referred to [41, 40] for details about unchanged features. Moreover, the correspondence principle is used to take shortcuts when the whole proof does not bring anything new. 
The methodology followed here is in line with our previous works on interface homogenisation and auxiliary field formalism. However, taking attenuation from basis into account is not incremental. It is instructive to compare the effective coefficient without attenuation (11), obtained in [31], and the new coefficient (22). The auxiliary fields are then largely modified, which is the subject of sections 3.4 and 4.3. The paper is then organized as follows:

- In Section 2, the physical problem under study is first presented and the homogenized model of a thin resonant microstructure without dissipation in the inclusions is then recalled.

- The homogenized interface conditions in the dissipative case are derived in Section 3. A planewave exact solution is computed. An energy balance is stated. Auxiliary fields are introduced along the interface, leading to local-in-time jump conditions.

- Section 4 recalls the numerical methods used for the time-domain simulations: the ADER-4 scheme is implemented to integrate the evolution equations [24], and the ESIM discretizes the resonant jump conditions on a uniform Cartesian grid [22, 21]. This presentation essentially emphasizes the differences with the inviscid case. The focus is put on the computation of the auxiliary fields, which is largely modified compared with the inviscid case.

- Numerical experiments are proposed in Section 5. Comparisons between numerical and semianalytical solutions of the homogenized problem validate the auxiliary-field approach. The relevance of homogenization is assessed by comparing microstructured and homogenized fields. Lastly, a critical value of the dissipation parameter is found, which maximises the absorption of the waves at a frequency close to one resonant frequency. When the interface is illuminated simultaneously from both sides and at this frequency, Coherent Perfect Absorption is reached and the simulations show very clearly that the waves are totally absorbed by the interface.

- Section 6 concludes the article and draws future lines of research. Technical details about the discretization of interfaces are given in Appendix A.

\section{Preliminaries}

\subsection{Microstructured configuration}

Problem statement. Let us consider the 2D propagation of anti-plane shear waves across a periodic row of inclusions $\cup_{i} \Omega_{i}$ embedded within a homogeneous matrix $\Omega_{m}$. Both media are isotropic, whereas damping is considered only in the inclusions to model the overall dissipation induced by bonding with the matrix, boundary layers, etc. Depending on the solid under consideration, it may be relevant to model the energy dissipation by a Maxwell, Kelvin-Voigt, standard solid or more sophisticated model $[16,19]$. However in this paper, our goal is not to describe precisely the dissipative process for a given medium. The questions we address are of methodological nature, and thus it is not relevant to privilege one viscoelasticity model over another. Consequently, we find it more relevant to consider a structural dissipation model, where damping is introduced through the equation of momentum.

The thickness and the periodicity of the row are denoted by $e$ and $h$, respectively, with $e=\mathcal{O}(h)$. The time and the spatial coordinates are denoted by $t$ and $\boldsymbol{X}=\left(X_{1}, X_{2}\right)$ with $X_{2}$ being the direction of periodicity of the inclusions, as shown in Figure 1. The physical parameters of the microstructured medium are the mass density $\rho_{h}$, the shear modulus $\mu_{h}$ and the dissipation parameter $\gamma_{h}$. The latter 


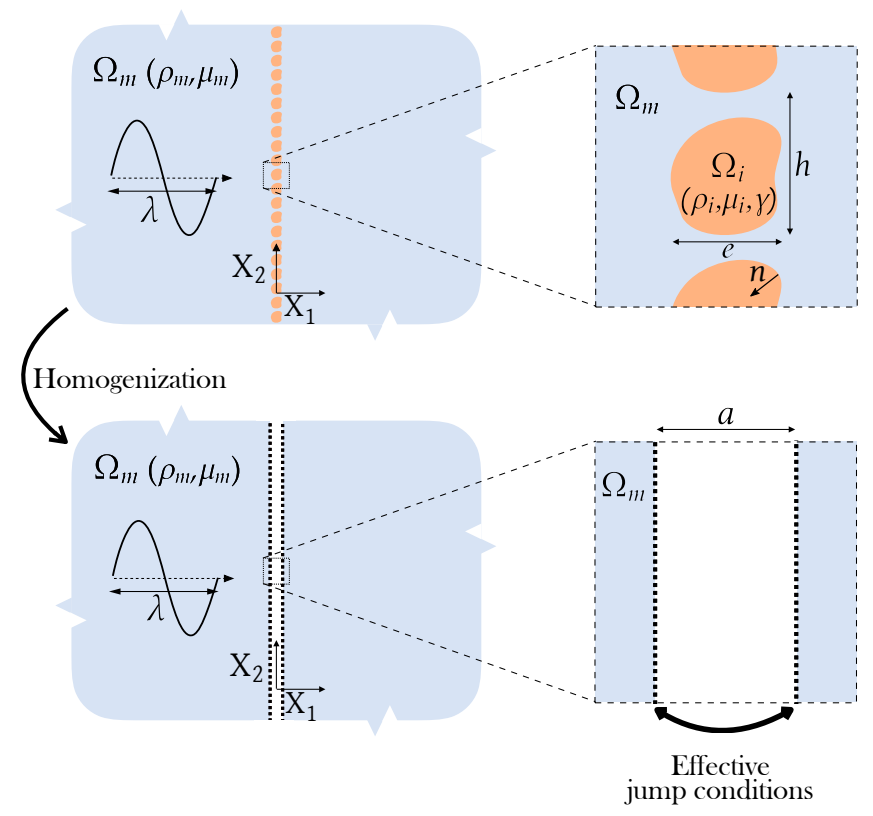

Figure 1: Homogenization process for a single periodic row of inclusions.

is inverse proportional to the damping, so that $\gamma_{h}=+\infty$ corresponds to an inviscid medium. The parameters are positive and piecewise constant:

$$
\left(\rho_{h}, \mu_{h}, \gamma_{h}\right)(\boldsymbol{X})= \begin{cases}\left(\rho_{m}, \mu_{m},+\infty\right) & \text { in the matrix, } \\ \left(\rho_{i}, \mu_{i}, \gamma\right) & \text { in the inclusions. }\end{cases}
$$

We introduce the scalar velocity field $V_{h}$ and the stress vector field $\Sigma_{h}=\left(\Sigma_{1 h}, \Sigma_{2 h}\right)^{\top}$. Evolution equations of linear anti-plane elasticity write as the first-order system:

$$
\left\{\begin{array}{l}
\partial_{t} \boldsymbol{\Sigma}_{h}(\boldsymbol{X}, t)=\mu_{h} \boldsymbol{\nabla} V_{h}(\boldsymbol{X}, t), \\
\rho_{h} \partial_{t} V_{h}(\boldsymbol{X}, t)+\frac{1}{\gamma_{h}} V_{h}(\boldsymbol{X}, t)=\operatorname{div} \boldsymbol{\Sigma}_{h}(\boldsymbol{X}, t),
\end{array}\right.
$$

with $V_{h}$ and $\boldsymbol{\Sigma}_{h} \cdot \boldsymbol{n}$ being continuous at each matrix/inclusion interface $\partial \Omega_{i}$, given that $\boldsymbol{n}$ is the inward unit normal on each $\partial \Omega_{i}$. This system is also relevant to the acoustic framework for which $\Sigma_{h}, V_{h}, \rho_{h}$ and $1 / \mu_{h}$ would stand instead for the velocity, pressure, compressibility and mass density, respectively. For all time $t \geq 0$, one defines

$$
\left\{\begin{array}{l}
\mathcal{E}_{h}(t)=\frac{1}{2} \int_{\mathcal{D}}\left\{\frac{1}{\mu_{h}(\boldsymbol{X})}\left(\boldsymbol{\Sigma}_{h}(\boldsymbol{X}, t)\right)^{2}+\rho_{h}(\boldsymbol{X})\left(V_{h}(\boldsymbol{X}, t)\right)^{2}\right\} \mathrm{d} \boldsymbol{X}, \\
\Upsilon_{h}^{\gamma}(t)=\frac{1}{\gamma} \int_{\cup_{i} \Omega_{i}}\left(V_{h}(\boldsymbol{X}, t)\right)^{2} \mathrm{~d} \boldsymbol{X},
\end{array}\right.
$$

where $\mathcal{D}$ is a bounded domain chosen to be sufficiently large to neglect the boundary terms. Then the energy identity holds

$$
\frac{\mathrm{d}}{\mathrm{d} t} \mathcal{E}_{h}(t)=-\Upsilon_{h}^{\gamma}(t) \leq 0 .
$$

When $\gamma=+\infty$, the inviscid case is recovered and the energy is conserved. 
Regime of study. The wavelength $\lambda_{m}$ within the matrix is assumed to be much larger than the spacing $h$ between the inclusions. Defining the wavenumber within the matrix as $k_{m}=2 \pi / \lambda_{m}$, we introduce $\eta=k_{m} h$ that satisfies $\eta \ll 1$ for the configurations of interest. Local resonances can occur when the wavelength within an inclusion is of the order of $h$ [2]: $k_{i} h=\mathcal{O}(1)$, with $k_{i}=\omega \sqrt{\rho_{i} / \mu_{i}}$. In particular, this condition is satisfied for a low contrast in mass density $\rho_{i} / \rho_{m}=\mathcal{O}(1)$ and a high contrast in shear modulus $\mu_{i} / \mu_{m}=\mathcal{O}\left(\eta^{2}\right)$, a configuration which we consider in the present study. These geometrical and material assumptions are summarized as follows:

Assumption 1. $\eta=k_{m} h \ll 1$ while $\rho_{i} / \rho_{m}=\mathcal{O}(1)$ and $\mu_{i} / \mu_{m}=\mathcal{O}\left(\eta^{2}\right)$.

Two-scale homogenization techniques and matched-asymptotic expansions can be applied to the microstructured problem defined above. They involve five steps: (i) two space coordinates are introduced to describe both the slow variations and the small-scale fast variations of the fields; (ii) the fields are expanded using the ansatz of a two-scale asymptotic expansion; (iii) matching conditions between the far field and the near field are formulated in an intermediate region close to the inclusions; (iv) these matching conditions identified at orders $\mathcal{O}(1)$ and $\mathcal{O}(\eta)$ provide the jump conditions for the effective fields at order $\mathcal{O}\left(\eta^{2}\right)$; (v) the interface of zero thickness on which the jump conditions apply is then replaced by an equivalent enlarged interface of thickness $a=\mathcal{O}(h)$, as sketched in Figure 1. This enlargement is needed for stability purpose.

Some notations are introduced. The fast scale of coordinates is $\boldsymbol{y}=\boldsymbol{X} / h=\left(y_{1}, y_{2}\right)^{\top}$. The domain $\Omega$ is the elementary cell $\mathbb{R} \times[-1 / 2,1 / 2]$ in $\boldsymbol{y}$-coordinates that contains one single inclusion $\Omega_{i}$, and $\left(\boldsymbol{e}_{1}, \boldsymbol{e}_{2}\right)$ is the canonical basis of $\mathbb{R}^{2}$. The Fourier transform in time of a function $g(t)$ is defined by:

$$
\mathscr{F}[g](\omega)=\hat{g}(\omega)=\int_{\mathbb{R}} g(t) e^{-i \omega t} \mathrm{~d} t
$$

where $\omega$ is the angular frequency. Lastly, the jump and the mean value of a field $h(\boldsymbol{X})$ at the enlarged interface are denoted by

$$
\llbracket h(\boldsymbol{X}) \rrbracket_{a}=h\left(a / 2, X_{2}\right)-h\left(-a / 2, X_{2}\right), \quad\langle h(\boldsymbol{X})\rangle_{a}=\frac{1}{2}\left(h\left(a / 2, X_{2}\right)+h\left(-a / 2, X_{2}\right)\right) .
$$

Here we recall the known results in the inviscid case. In Section 3, we will adapt them to the damped case. In each case, the findings are presented in the frequency domain, then in the time domain, which allows to state an energy balance.

\subsection{Known case: inviscid configuration}

Frequency-domain formulation. The case $\gamma=+\infty$ has been investigated in [31]. At order $\mathcal{O}\left(\eta^{2}\right)$, the homogenized fields $V$ and $\Sigma=\left(\Sigma_{1}, \Sigma_{2}\right)^{\top}$ satisfy in the frequency domain:

$$
\left\{\begin{array}{lr}
\mathrm{i} \omega \hat{\boldsymbol{\Sigma}}(\boldsymbol{X}, \omega)=\mu_{m} \boldsymbol{\nabla} \hat{V}(\boldsymbol{X}, \omega) & \left(\left|X_{1}\right| \geq a / 2, Y \in \mathbb{R}\right) \\
\mathrm{i} \omega \rho_{m} \hat{V}(\boldsymbol{X}, \omega)=\operatorname{div} \hat{\boldsymbol{\Sigma}}(\boldsymbol{X}, \omega) & \left(\left|X_{1}\right| \geq a / 2, Y \in \mathbb{R}\right) \\
\llbracket \hat{V} \rrbracket_{a}=h\left\{B\left\langle\partial_{X_{1}} \hat{V}\right\rangle_{a}+B_{2}\left\langle\partial_{X_{2}} \hat{V}\right\rangle_{a}\right\} & \left(X_{2} \in \mathbb{R}\right) \\
\llbracket \hat{\Sigma}_{1} \rrbracket_{a}=h\left\{S\left\langle\partial_{X_{1}} \hat{\Sigma}_{1}\right\rangle_{a}+C_{1}\left\langle\partial_{X_{2}} \hat{\Sigma}_{1}\right\rangle_{a}+C_{2}\left\langle\partial_{X_{2}} \hat{\Sigma}_{2}\right\rangle_{a}+\mathscr{D}_{\infty}(\omega)\langle\operatorname{div} \hat{\boldsymbol{\Sigma}}\rangle_{a}\right\} & \left(X_{2} \in \mathbb{R}\right),
\end{array}\right.
$$


where the effective coefficients $B, B_{2}, S, C_{1}, C_{2}$ and $\mathscr{D}_{\infty}(\omega)$ are computed as follows. First, let define $\varphi \in] 0,1[$ such that $e \varphi / h$ is the surface of the inclusion in $\boldsymbol{y}$-coordinates. Then $S$ writes

$$
S=\frac{a}{h}-\frac{e \varphi}{h} .
$$

Secondly, let us introduce the cell problems $(j=1,2)$ :

$$
\begin{cases}\Delta_{\boldsymbol{y}} \Phi^{(j)}(\boldsymbol{y})=0 & \left(\boldsymbol{y} \in \Omega \backslash \Omega_{i}\right), \\ {\left[\boldsymbol{\nabla}_{\boldsymbol{y}} \Phi^{(j)}(\boldsymbol{y})+\boldsymbol{e}_{j}\right] \cdot \boldsymbol{n}=0} & \left(\boldsymbol{y} \in \partial \Omega_{i}\right), \\ \lim _{y_{1} \rightarrow \pm \infty} \boldsymbol{\nabla}_{\boldsymbol{y}} \Phi^{(j)}\left(y_{1}, y_{2}\right)=\mathbf{0}, & \end{cases}
$$

where the cell functions $\Phi^{(j)}(\boldsymbol{y})$ are $y_{2}$-periodic. Then $B, B_{2}, C_{1}$ and $C_{2}$ write

$$
\begin{aligned}
& B_{j}=\lim _{y_{1} \rightarrow+\infty}\left[\Phi^{(j)}\left(y_{1}, y_{2}\right)-\Phi^{(j)}\left(-y_{1}, y_{2}\right)\right], \quad B=\frac{a}{h}+B_{1}, \\
& C_{j}=-\int_{\Omega \backslash \Omega_{i}} \nabla \Phi^{(j)}(\boldsymbol{y}) \cdot \boldsymbol{e}_{2} \mathrm{~d} \boldsymbol{y} .
\end{aligned}
$$

The effective coefficients $B, B_{2}, S, C_{1}$ and $C_{2}$ depend on the geometry of the inclusions but not on their physical properties, contrary to the non-resonant case [27]. Third and last, one introduces the Dirichlet problem:

$$
\begin{cases}\Delta_{\boldsymbol{y}} \psi_{\infty}(\boldsymbol{y}, \omega)+\kappa_{\infty}^{2}(\omega) \psi_{\infty}(\boldsymbol{y}, \omega)=0 & \left(\boldsymbol{y} \in \Omega_{i}\right) \\ \psi_{\infty}(\boldsymbol{y}, \omega)=1 & \left(\boldsymbol{y} \in \partial \Omega_{i}\right)\end{cases}
$$

with

$$
\kappa_{\infty}^{2}(\omega)=\frac{\rho_{i} h^{2}}{\mu_{i}} \omega^{2}
$$

Then the frequency-dependent coefficient $\mathscr{D}_{\infty}(\omega)$ in $(5)$ is

$$
\mathscr{D}_{\infty}(\omega)=\frac{\rho_{i}}{\rho_{m}} \int_{\Omega_{i}} \psi_{\infty}(\boldsymbol{y}, \omega) \mathrm{d} \boldsymbol{y}
$$

The explicit dependency of $\mathscr{D}_{\infty}$ on the frequency is reached through a modal expansion of $\psi_{\infty}$ on the basis of the eigensystems $\left(\lambda_{r}, P_{r}\right)_{r e \geq 1}$ that is associated with the following self-adjoint eigenvalue problem within the inclusion:

$$
\begin{cases}\Delta_{\boldsymbol{y}} P_{r}(\boldsymbol{y})+\lambda_{r} P_{r}(\boldsymbol{y})=0 & \left(\boldsymbol{y} \in \Omega_{i}\right) \\ P_{r}(\boldsymbol{y})=0 & \left(\boldsymbol{y} \in \partial \Omega_{i}\right)\end{cases}
$$

Let us define the real-valued coefficients $\left\{\alpha_{r}\right\}_{r \geq 0}$ and the resonant frequencies $\left\{\omega_{r}\right\}_{r \geq 1}$ :

$$
\alpha_{0}=\frac{\rho_{i}}{\rho_{m}} \int_{\Omega_{i}} \mathrm{~d} \boldsymbol{y} \quad \alpha_{r}=\sqrt{\frac{\rho_{i}}{\rho_{m}}} \int_{\Omega_{i}} P_{r}(\boldsymbol{y}) \mathrm{d} \boldsymbol{y} \quad \text { and } \quad \omega_{r}=\frac{1}{h} \sqrt{\frac{\mu_{i}}{\rho_{i}} \lambda_{r}} .
$$

Then after some calculations, the frequency-dependent coefficient $\mathscr{D}_{\infty}(\omega)$ in (5) and (11) can be recast as the infinite series

$$
\mathscr{D}_{\infty}(\omega)=\alpha_{0}-\sum_{r \geq 1} \alpha_{r}^{2} \frac{\omega^{2}}{\omega^{2}-\omega_{r}^{2}}
$$


The coefficient $\mathscr{D}_{\infty}$ encapsulates the parameters of inclusions as well as their geometries. In practice, the infinite series is truncated to a finite number $N_{R}$ of resonances. As seen in (13), the resonances associated with null-mean modes do not contribute to the effective model obtained. It constitutes a limitation of the second-order homogenized model, already noted in [31]. The effect of this failure has been shown to be negligible in [41].

Time-domain formulation. Let us define the inner problem:

$$
\begin{cases}\partial_{t} \boldsymbol{\Sigma}_{i}\left(\boldsymbol{y}, X_{2}, t\right)=\frac{\mu_{i}}{h} \boldsymbol{\nabla}_{\boldsymbol{y}} W_{i}\left(\boldsymbol{y}, X_{2}, t\right) & \left(\boldsymbol{y} \in \Omega_{i}\right), \\ \rho_{i} \partial_{t} W_{i}\left(\boldsymbol{y}, X_{2}, t\right)=\frac{1}{h} \operatorname{div}_{\boldsymbol{y}} \boldsymbol{\Sigma}_{i}\left(\boldsymbol{y}, X_{2}, t\right) & \left(\boldsymbol{y} \in \Omega_{i}\right), \\ W_{i}\left(\boldsymbol{y}, X_{2}, t\right)=\left\langle V\left(\cdot, X_{2}, t\right)\right\rangle_{a} & \left(\boldsymbol{y} \in \partial \Omega_{i}\right),\end{cases}
$$

where the field $W_{i}$ is a function of $X_{2}$ through the prescribed boundary condition $\left\langle V\left(\cdot, X_{2}, t\right)\right\rangle_{a}$. The repeated index $i$ in (15) denotes the inclusion and must not be confused with Einstein's notation. In [41], it has been proven that

$$
\begin{aligned}
\mathrm{i} \omega \rho_{i} \int_{\Omega_{i}} \hat{W}_{i} \mathrm{~d} \boldsymbol{y} & =\frac{\rho_{i}}{\rho_{m}} \int_{\Omega_{i}} \psi_{\infty}(\boldsymbol{y}, \omega) \mathrm{d} \boldsymbol{y}\langle\operatorname{div} \hat{\boldsymbol{\Sigma}}\rangle_{a} \\
& =\mathscr{D}_{\infty}(\omega)\langle\operatorname{div} \hat{\boldsymbol{\Sigma}}\rangle_{a} .
\end{aligned}
$$

Inverse Fourier transforms of (5) and (16) yield the homogenized model in the time domain:

$$
\left\{\begin{array}{rlrl}
\partial_{t} \boldsymbol{\Sigma} & =\mu_{m} \nabla V & & \left(\left|X_{1}\right| \geq a / 2, X_{2} \in \mathbb{R}\right) \\
\partial_{t} V & =\frac{1}{\rho_{m}} \operatorname{div} \boldsymbol{\Sigma} & & \left(\left|X_{1}\right| \geq a / 2, X_{2} \in \mathbb{R}\right) \\
\llbracket V \rrbracket_{a} & =h\left\{B\left\langle\partial_{X_{1}} V\right\rangle_{a}+B_{2}\left\langle\partial_{X_{2}} V\right\rangle_{a}\right\} & \left(X_{2} \in \mathbb{R}\right) \\
\llbracket \Sigma_{1} \rrbracket_{a}=h\left\{S\left\langle\partial_{X_{1}} \Sigma_{1}\right\rangle_{a}+C_{1}\left\langle\partial_{X_{2}} \Sigma_{1}\right\rangle_{a}+C_{2}\left\langle\partial_{X_{2}} \Sigma_{2}\right\rangle_{a}+\rho_{i} \int_{\Omega_{i}} \partial_{t} W_{i} \mathrm{~d} \boldsymbol{y}\right\} & \left(X_{2} \in \mathbb{R}\right) .
\end{array}\right.
$$

An energy balance can be deduced from (17). A bounded domain $\mathcal{D}$ is chosen to be sufficiently

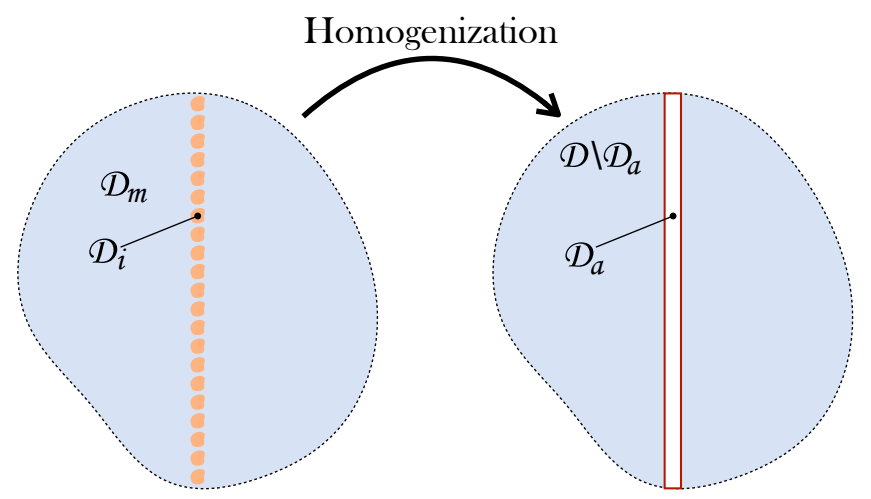

Figure 2: (left) Domain $\mathcal{D}=\cup_{i} \mathcal{D}_{i} \cup \mathcal{D}_{m}$ considered for the energy analysis in the original microstructured configuration, (right) domain $\mathcal{D} \backslash \mathcal{D}_{a}$ for the analysis of the effective problem. 
large to neglect the boundary terms, $\mathcal{D}_{a}$ is the intersection between $\mathcal{D}$ and the enlarged interface, and $\mathcal{I}=\left\{X_{2}: \boldsymbol{X} \in \mathcal{D}_{a}\right\}$, as sketched in Figure 2. Then one introduces $\mathcal{E}_{b}$ and $\mathcal{E}_{i}$ which are bulk and interface terms, respectively:

$$
\begin{aligned}
& \mathcal{E}_{b}=\frac{1}{2} \int_{\mathcal{D} \backslash \mathcal{D}_{a}}\left\{\frac{1}{\mu_{m}}(\boldsymbol{\Sigma})^{2}+\rho_{m}(V)^{2}\right\} \mathrm{d} \boldsymbol{X}, \\
& \mathcal{E}_{i}=\frac{h}{2} \int_{\mathcal{I}}\left\{S \rho_{m}\langle V\rangle_{a}^{2}+\frac{B}{\mu_{m}}\left\langle\Sigma_{1}\right\rangle_{a}^{2}+\frac{S-C_{2}}{\mu_{m}}\left\langle\Sigma_{2}\right\rangle_{a}^{2}+\int_{\Omega_{i}}\left(\rho_{i} W_{i}^{2}+\frac{1}{\mu_{i}}\left|\boldsymbol{\Sigma}_{i}\right|^{2}\right) \mathrm{d} \boldsymbol{y}\right\} \mathrm{d} X_{2} .
\end{aligned}
$$

In the Section 2.2 of [41], it has been proven that the $\mathcal{E}=\mathcal{E}_{b}+\mathcal{E}_{i}$ is constant over time. It has also be proven that $a \geq e$ implies $\mathcal{E}_{i} \geq 0$, so that $\mathcal{E}$ is an energy, which ensures the stability of the homogenized problem.

\section{Homogenized resonant model with dissipation}

\subsection{Frequency-domain formulation}

The homogenization steps followed in [31] can be customary extended to the case $\gamma \neq+\infty$. For the sake of brevity, the full derivation is not detailed here, since the findings can be obtained using the correspondence principle: see e.g. chapter 3 of [4]. The microstructured problem (1) can be recast in the frequency domain as

$$
\left\{\begin{array}{l}
\mathrm{i} \omega \hat{\boldsymbol{\Sigma}}_{h}(\boldsymbol{X}, \omega)=\mu_{h} \boldsymbol{\nabla} \hat{V}_{h}(\boldsymbol{X}, \omega) \\
\mathrm{i} \omega \hat{\rho}_{h}^{\gamma} \hat{V}_{h}(\boldsymbol{X}, \omega)=\operatorname{div} \hat{\boldsymbol{\Sigma}}_{h}(\boldsymbol{X}, \omega)
\end{array}\right.
$$

with

$$
\hat{\rho}_{h}^{\gamma}(\boldsymbol{X})= \begin{cases}\rho_{m} & \text { in the matrix, } \\ \rho_{i}\left(1+\frac{1}{\mathrm{i} \omega \gamma \rho_{i}}\right) & \text { in the inclusions, }\end{cases}
$$

which is exactly the same frequency-domain formulation than in the inviscid case. Consequently, one expects to find the same effective jump conditions (5) by replacing the term $\rho_{i}$ by $\rho_{i}\left(1+\frac{1}{\mathrm{i} \omega \gamma \rho_{i}}\right)$ in the effective coefficients. The coefficients $S, B, B_{2}, C_{1}$ and $C_{2}$ do not depend on $\rho_{i}$, thus they are unchanged. On the contrary, $\mathscr{D}_{\infty}(\omega)$ depends implicitly on $\rho_{i}$, and thus it is changed into $\mathscr{D}_{\gamma}(\omega)$, leading to the new homogenized model with damping:

$$
\left\{\begin{array}{lr}
\operatorname{i\omega } \hat{\Sigma}(\boldsymbol{X}, \omega)=\mu_{m} \nabla \hat{V}(\boldsymbol{X}, \omega) & \left(\left|X_{1}\right| \geq a / 2, X_{2} \in \mathbb{R}\right) \\
\mathrm{i} \omega \rho_{m} \hat{V}(\boldsymbol{X}, \omega)=\operatorname{div} \hat{\boldsymbol{\Sigma}}(\boldsymbol{X}, \omega) & \left(\left|X_{1}\right| \geq a / 2, X_{2} \in \mathbb{R}\right) \\
\llbracket \hat{V} \|_{a}=h\left\{B\left\langle\partial_{X_{1}} \hat{V}\right\rangle_{a}+B_{2}\left\langle\partial_{X_{2}} \hat{V}\right\rangle_{a}\right\} & \left(X_{2} \in \mathbb{R}\right) \\
\llbracket \hat{\Sigma}_{1} \rrbracket_{a}=h\left\{S\left\langle\partial_{X_{1}} \hat{\Sigma}_{1}\right\rangle_{a}+C_{1}\left\langle\partial_{X_{2}} \hat{\Sigma}_{1}\right\rangle_{a}+C_{2}\left\langle\partial_{X_{2}} \hat{\Sigma}_{2}\right\rangle_{a}+\mathscr{D}_{\gamma}(\omega)\langle\operatorname{div} \hat{\boldsymbol{\Sigma}}\rangle_{a}\right\} & \left(X_{2} \in \mathbb{R}\right) .
\end{array}\right.
$$

The new effective coefficient $\mathscr{D}_{\gamma}(\omega)$ is obtained as follows. Replacing $\rho_{i}$ by $\rho_{i}\left(1+\frac{1}{\mathrm{i} \omega \gamma \rho_{i}}\right)$, the Dirichlet problem (9) becomes

$$
\begin{cases}\Delta_{\boldsymbol{y}} \psi_{\gamma}(\boldsymbol{y}, \omega)+\kappa_{\gamma}^{2}(\omega) \psi_{\gamma}(\boldsymbol{y}, \omega)=0 & \left(\boldsymbol{y} \in \Omega_{i}\right), \\ \psi_{\gamma}(\boldsymbol{y}, \omega)=1 & \left(\boldsymbol{y} \in \partial \Omega_{i}\right),\end{cases}
$$


where (10) is replaced by

$$
\kappa_{\gamma}^{2}(\omega)=\frac{\rho_{i} h^{2}}{\mu_{i}} \omega^{2}\left(1+\frac{1}{i \omega \gamma \rho_{i}}\right) .
$$

Then the frequency-dependent coefficient in (11) becomes

$$
\mathscr{D}_{\gamma}(\omega)=\frac{\rho_{i}}{\rho_{m}}\left(1+\frac{1}{\mathrm{i} \omega \gamma \rho_{i}}\right) \int_{\Omega_{i}} \psi_{\gamma}(\boldsymbol{y}, \omega) \mathrm{d} \boldsymbol{y}
$$

Keeping the same definitions of $\alpha_{0}, \alpha_{r \geq 1}$ and $\omega_{r}$ than in (13), the modal expansion of (22) yields

$$
\mathscr{D}_{\gamma}(\omega)=\left(1+\frac{1}{\mathrm{i} \omega \gamma \rho_{i}}\right)\left\{\alpha_{0}-\sum_{r \geq 1} \alpha_{r}^{2} \frac{\omega^{2}-\frac{\mathrm{i} \omega}{\gamma \rho_{i}}}{\omega^{2}-\frac{\mathrm{i} \omega}{\gamma \rho_{i}}-\omega_{r}^{2}}\right\} .
$$

The frequency-dependent effective coefficient (23) recovers (14) in the limit-case $\gamma=+\infty$, but it does not appear as a trivial extension of the inviscid configuration. As in (14), the infinite series is truncated to a finite number $N_{R}$ of resonances, and the null-mean modes lead to $\alpha_{r}=0$, so that they do not contribute to the effective model obtained. The effect of this limitation will be examined numerically in Section 5.

\subsection{Plane-wave solution}

Let us consider an harmonic plane wave impacting with an angle $\theta$ the enlarged interface located between $X_{1}=-a / 2$ and $X_{1}=+a / 2$. The wavefield solution $\hat{\boldsymbol{u}}=\left(\hat{V}, \hat{\Sigma}_{1}, \hat{\Sigma}_{2}\right)^{\top}$ is decomposed into incident $\hat{\boldsymbol{u}}_{I}$, reflected $\hat{\boldsymbol{u}}_{R}$ and transmitted $\hat{\boldsymbol{u}}_{T}$ plane waves, ie $\hat{\boldsymbol{u}}=\hat{\boldsymbol{u}}_{I}+\hat{\boldsymbol{u}}_{R}+\hat{\boldsymbol{u}}_{T}$, that write

$$
\left\{\begin{aligned}
& \hat{\boldsymbol{u}}_{I}=\left(\begin{array}{c}
1 / \mu_{m} \\
-\cos \theta / c_{m} \\
-\sin \theta / c_{m}
\end{array}\right) \exp \left(-\mathrm{i} \boldsymbol{k}_{I} \cdot \boldsymbol{X}\right) \\
& \hat{\boldsymbol{u}}_{R}=\left(\begin{array}{c}
1 / \mu_{m} \\
+\cos \theta / c_{m} \\
-\sin \theta / c_{m}
\end{array}\right) \exp \left(-\mathrm{i} \boldsymbol{k}_{R} \cdot \boldsymbol{X}\right) \mathcal{R}(\omega), \\
& \hat{\boldsymbol{u}}_{T}=\left(\begin{array}{c}
1 / \mu_{m} \\
-\cos \theta / c_{m} \\
-\sin \theta / c_{m}
\end{array}\right) \exp \left(-\mathrm{i} \boldsymbol{k}_{T} \cdot \boldsymbol{X}\right) \mathcal{T}(\omega) .
\end{aligned}\right.
$$

The wavevectors $\boldsymbol{k}_{I}, \boldsymbol{k}_{R}$ and $\boldsymbol{k}_{T}$ of norm $\omega / c_{m}$ are normal to the wave fronts. Using the jump conditions in (20) yields a $2 \times 2$ system satisfied by the reflection coefficient $\mathcal{R}$ and the transmission coefficient $\mathcal{T}$, whose solution is

$$
\left\{\begin{array}{l}
\mathcal{R}(\omega)=\frac{i \omega \mathcal{L}(\omega)}{2 Z+i \omega \mathcal{N}(\omega)-\omega^{2} \mathcal{M}(\omega)} \exp \left(i \frac{\omega}{c_{m}} a \cos \theta\right) \\
\mathcal{T}(\omega)=\frac{2 Z+i \omega \mathcal{G}+\omega^{2} \mathcal{M}(\omega)}{2 Z+i \omega \mathcal{N}(\omega)-\omega^{2} \mathcal{M}(\omega)} \exp \left(i \frac{\omega}{c_{m}} a \cos \theta\right)
\end{array}\right.
$$


The coefficients in (25) are

$$
\left\{\begin{array}{l}
\mathcal{L}(\omega)=h\left((-S+B) \cos (\theta)^{2}-C_{2} \sin (\theta)^{2}+\left(B_{2}-C_{1}\right) \cos (\theta) \sin (\theta)-\mathscr{D}_{\gamma}(\omega)\right), \\
\mathcal{G}=-h\left(C_{1}+B_{2}\right) \cos (\theta) \sin (\theta) \\
Z=c_{m} \cos \theta \\
\mathcal{N}(\omega)=h\left(S \cos (\theta)^{2}+C_{2} \sin (\theta)^{2}+B \cos (\theta)^{2}+\mathscr{D}_{\gamma}(\omega)\right) \\
\mathcal{M}(\omega)=\frac{h^{2}}{2 c_{m}}\left(B S \cos (\theta)^{2}-B_{2} C_{1} \sin (\theta)^{2}+B C_{2} \sin (\theta)^{2}+B \mathscr{D}_{\gamma}(\omega)\right) \cos (\theta) .
\end{array}\right.
$$

The frequency-dependent effective coefficient (23) is involved in the exact solution through $\mathcal{L}(\omega)$, $\mathcal{M}(\omega)$ and $\mathcal{N}(\omega)$ in (25)-(26). The effect of damping on $\mathcal{R}$ and $\mathcal{T}$ will be examined numerically in Section 5 .

\subsection{Time-domain formulation}

Replacing $\rho_{i}$ by $\rho_{i}\left(1+\frac{1}{\mathrm{i} \omega \gamma \rho_{i}}\right)$ in (15) yields the inner problem in the frequency domain:

$$
\begin{cases}\mathrm{i} \omega \hat{\boldsymbol{\Sigma}}_{i}\left(\boldsymbol{y}, X_{2}, t\right)=\frac{\mu_{i}}{h} \boldsymbol{\nabla}_{\boldsymbol{y}} \hat{W}_{i}\left(\boldsymbol{y}, X_{2}, t\right) & \left(\boldsymbol{y} \in \Omega_{i}\right), \\ \rho_{i}\left(1+\frac{1}{\mathrm{i} \omega \gamma \rho_{i}}\right) \hat{W}_{i}\left(\boldsymbol{y}, X_{2}, t\right)=\frac{1}{h} \operatorname{div}_{\boldsymbol{y}} \hat{\boldsymbol{\Sigma}}_{i}\left(\boldsymbol{y}, X_{2}, t\right) & \left(\boldsymbol{y} \in \Omega_{i}\right), \\ \hat{W}_{i}\left(\boldsymbol{y}, X_{2}, t\right)=\left\langle\hat{V}\left(\cdot, X_{2}, t\right)\right\rangle_{a} & \left(\boldsymbol{y} \in \partial \Omega_{i}\right) .\end{cases}
$$

Similarly, (16) becomes

$$
\begin{aligned}
\mathrm{i} \omega \rho_{i}\left(1+\frac{1}{\mathrm{i} \omega \gamma \rho_{i}}\right) \int_{\Omega_{i}} \hat{W}_{i} \mathrm{~d} \boldsymbol{y} & =\frac{\rho_{i}}{\rho_{m}}\left(1+\frac{1}{\mathrm{i} \omega \gamma \rho_{i}}\right) \int_{\Omega_{i}} \psi_{\gamma}(\boldsymbol{y}, \omega) \mathrm{d} \boldsymbol{y}\langle\operatorname{div} \hat{\boldsymbol{\Sigma}}\rangle_{a} \\
& =\mathscr{D}_{\gamma}(\omega)\langle\operatorname{div} \hat{\boldsymbol{\Sigma}}\rangle_{a} .
\end{aligned}
$$

Inverse Fourier transforms of (20), (27) and (28) yield the homogenized model in the time domain:

$$
\left\{\begin{array}{rlrl}
\partial_{t} \boldsymbol{\Sigma} & =\mu_{m} \nabla V & \left(\left|X_{1}\right| \geq a / 2, X_{2} \in \mathbb{R}\right) \\
\partial_{t} V & =\frac{1}{\rho_{m}} \operatorname{div} \boldsymbol{\Sigma} & \left(\left|X_{1}\right| \geq a / 2, X_{2} \in \mathbb{R}\right) \\
\llbracket V \rrbracket_{a}=h\left\{B\left\langle\partial_{X_{1}} V\right\rangle_{a}+B_{2}\left\langle\partial_{X_{2}} V\right\rangle_{a}\right\} & \left(X_{2} \in \mathbb{R}\right) \\
\llbracket \Sigma_{1} \rrbracket_{a}=h\left\{S\left\langle\partial_{X_{1}} \Sigma_{1}\right\rangle_{a}+C_{1}\left\langle\partial_{X_{2}} \Sigma_{1}\right\rangle_{a}+C_{2}\left\langle\partial_{X_{2}} \Sigma_{2}\right\rangle_{a}+\rho_{i} \int_{\Omega_{i}} \partial_{t} W_{i} \mathrm{~d} \boldsymbol{y}+\frac{1}{\gamma} \int_{\Omega_{i}} W_{i} \mathrm{~d} \boldsymbol{y}\right\} & \left(X_{2} \in \mathbb{R}\right),
\end{array}\right.
$$

where $W_{i}$ is solution in each inclusion of the inner problem:

$$
\begin{cases}\partial_{t} \boldsymbol{\Sigma}_{i}\left(\boldsymbol{y}, X_{2}, t\right)=\frac{\mu_{i}}{h} \boldsymbol{\nabla}_{\boldsymbol{y}} W_{i}\left(\boldsymbol{y}, X_{2}, t\right) & \left(\boldsymbol{y} \in \Omega_{i}\right), \\ \rho_{i} \partial_{t} W_{i}\left(\boldsymbol{y}, X_{2}, t\right)+\frac{1}{\gamma} W_{i}\left(\boldsymbol{y}, X_{2}, t\right)=\frac{1}{h} \operatorname{div}_{\boldsymbol{y}} \boldsymbol{\Sigma}_{i}\left(\boldsymbol{y}, X_{2}, t\right) & \left(\boldsymbol{y} \in \Omega_{i}\right), \\ W_{i}\left(\boldsymbol{y}, X_{2}, t\right)=\left\langle V\left(\cdot, X_{2}, t\right)\right\rangle_{a} & \left(\boldsymbol{y} \in \partial \Omega_{i}\right) .\end{cases}
$$

In the limit-case $\gamma=+\infty$, the equations (15) and (17) of the inviscid model are recovered. 
The energy balance can be calculated in the same way as in the case without dissipation. The expressions (18) are unchanged. The properties $B \geq 0$ and $S-C_{2} \geq 0$ if $a \geq e$ are independent of $\rho_{i}$ and are therefore always satisfied, so that $\mathcal{E}=\mathcal{E}_{b}+\mathcal{E}_{i}$ is an energy. On the other hand, the term $\gamma$ modifies the energy balance, which is written as follows

$$
\frac{\mathrm{d}}{\mathrm{d} t}\left(\mathcal{E}_{b}+\mathcal{E}_{i}\right)=-\Upsilon_{\gamma}
$$

with

$$
\Upsilon_{\gamma}=\frac{h}{\gamma} \int_{I} \int_{\Omega_{i}} W_{i}^{2} \mathrm{~d} \boldsymbol{y} \mathrm{d} X_{2} \geq 0 .
$$

This underlines that $\mathcal{E}$ is decreasing due to the dissipation.

\subsection{Formalism of auxiliary fields}

In (29), $W_{i}$ can be expressed as a time convolution with the Green's function in the inclusion domain $\Omega_{i}$. Handling numerically such a non-local-in-time term is very costly and would therefore reduce the computational gains associated with the homogenization. To circumvent this difficulty, the auxiliary variable approach is used. This formalism has been introduced in [40] to handle the resonant jump conditions without dissipation. Following the same method here, we introduce the auxiliary variables $\hat{J}_{r}$ and $\hat{G}_{r}$ associated with the resonance index $r \in\left\{1, \cdots, N_{R}\right\}$ and which satisfy:

$$
\left\{\begin{array}{l}
\left(\omega^{2}-\frac{1}{\gamma \rho_{i}} \mathrm{i} \omega-\omega_{r}^{2}\right) \hat{J}_{r}\left(X_{2}, \omega\right)=\alpha_{r}^{2}\left(1+\frac{1}{\gamma \rho_{i} \mathrm{i} \omega}\right)\left(\omega^{2}-\frac{1}{\gamma \rho_{i}} \mathrm{i} \omega\right)\langle\operatorname{div} \hat{\Sigma}\rangle_{a} \quad\left(X_{2} \in \mathbb{R}\right) . \\
\mathrm{i} \omega \hat{J}_{r}\left(X_{2}, \omega\right)=\hat{G}_{r}\left(X_{2}, \omega\right)
\end{array}\right.
$$

Combining (20), (23) and (33), a formal inverse Fourier transform yields the system

$$
\left\{\begin{array}{lr}
\partial_{t} \boldsymbol{\Sigma}(\boldsymbol{X}, t)=\mu_{m} \nabla V(\boldsymbol{X}, t) & \left(\left|X_{1}\right| \geq a / 2, X_{2} \in \mathbb{R}\right) \\
\partial_{t} V(\boldsymbol{X}, t)=\frac{1}{\rho_{m}} \operatorname{div} \boldsymbol{\Sigma}(\boldsymbol{X}, t) & \left(\left|X_{1}\right| \geq a / 2, X_{2} \in \mathbb{R}\right) \\
\partial_{t} J_{r}\left(X_{2}, t\right)=G_{r}\left(X_{2}, t\right) & \left(X_{2} \in \mathbb{R}, r=1, \ldots, N_{R}\right) \\
\partial_{t} G_{r}\left(X_{2}, t\right)=-\frac{1}{\gamma \rho_{i}} G_{r}\left(X_{2}, t\right)-\omega_{r}^{2} J_{r}\left(X_{2}, t\right)+\mu_{m} \alpha_{r}^{2}\left(\partial_{t}+\frac{2}{\gamma \rho_{i}}\right)\langle\Delta V\rangle_{a}+\left(\frac{\alpha_{r}}{\gamma \rho_{i}}\right)^{2}\langle\operatorname{div} \boldsymbol{\Sigma}\rangle_{a} \\
\llbracket V \rrbracket_{a}=\widetilde{B}_{1}\left\langle\partial_{X_{1}} V\right\rangle_{a}+\widetilde{B}_{2}\left\langle\partial_{X_{2}} V\right\rangle_{a} & \left(X_{2} \in \mathbb{R}\right) \\
\llbracket \Sigma_{1} \rrbracket_{a}=\widetilde{C}_{11}\left\langle\partial_{X_{1}} \Sigma_{1}\right\rangle_{a}+\widetilde{C}_{12}\left\langle\partial_{X_{2}} \Sigma_{1}\right\rangle_{a}+\widetilde{C}_{22}\left\langle\partial_{X_{2}} \Sigma_{2}\right\rangle_{a}+\widetilde{D}_{0}\langle V\rangle_{a}-h \sum_{r=1}^{N_{R}} J_{r} & \left(X_{2} \in \mathbb{R}\right),
\end{array}\right.
$$

where we have set $\widetilde{B}_{1}=h B, \widetilde{B}_{2}=h B_{2}, \widetilde{C}_{11}=h\left(S+\alpha_{0}\right), \widetilde{C}_{12}=h C_{1}, \widetilde{C}_{22}=h\left(C_{2}+\alpha_{0}\right)$ and $\widetilde{D}_{0}=\left(h \rho_{m} \alpha_{0}\right) /\left(\gamma \rho_{i}\right)$. When $\gamma=+\infty$, then equation (7) of [40] is recovered.

In (34), the evolution equation satisfied by $G_{r}$ involves $\partial_{t}\langle\Delta V\rangle_{a}$. This term could be replaced by $\langle\Delta \operatorname{div} \Sigma\rangle_{a}$ via conservation laws. However, it is preferable to leave it in this form for numerical purpose [40]. 


\section{Numerical modeling in 1D}

\section{1. $1 D$ setting}

In this section, we describe the numerical implementation of the system (34) in a one-dimensional configuration representative of the propagation of a plane wave illuminating a straight enlarged interface at normal incidence. In this case, the fields are independent from $X_{2}$ and the vector field $\boldsymbol{\Sigma}(\boldsymbol{X})$ reduces to the scalar field $\Sigma(X)=\Sigma_{1}\left(X_{1}\right)$, where $X=\boldsymbol{X} . \boldsymbol{e}_{1}$ and $\left(\boldsymbol{e}_{1}, \boldsymbol{e}_{2}\right)$ being the canonical basis of $\mathbb{R}^{2}$. The system (34) is recast for all $t$ as

$$
\begin{cases}\partial_{t} \Sigma(X, t)=\mu_{m} \partial_{X} V(X, t) & (|X| \geq a / 2) \\ \partial_{t} V(X, t)=\frac{1}{\rho_{m}} \partial_{X} \Sigma(X, t) & (|X| \geq a / 2) \\ \partial_{t} J_{r}(t)=G_{r}(t) & \left(r=1, \ldots, N_{R}\right) \\ \partial_{t} G_{r}(t)=-\frac{1}{\gamma \rho_{i}} G_{r}(t)-\omega_{r}^{2} J_{r}(t)+\mu_{m} \alpha_{r}^{2}\left(\partial_{t}+\frac{2}{\gamma \rho_{i}}\right)\left\langle\partial_{X X}^{2} V\right\rangle_{a}+\left(\frac{\alpha_{r}}{\gamma \rho_{i}}\right)^{2}\left\langle\partial_{X} \Sigma\right\rangle_{a} & \left(r=1, \ldots, N_{R}\right) \\ \llbracket V \rrbracket_{a}=\widetilde{B}_{1}\left\langle\partial_{X} V\right\rangle_{a} & \\ \llbracket \Sigma \rrbracket_{a}=\widetilde{C}_{11}\left\langle\partial_{X} \Sigma\right\rangle_{a}+\widetilde{D}_{0}\langle V\rangle_{a}-h \sum_{r=1}^{N_{R}} J_{r} . & \end{cases}
$$

As in [40], the solution $\boldsymbol{u}=(V, \Sigma)^{\top}$ of (35) is discretized using a uniform Cartesian grid with mesh size $\Delta X$ and time step $\Delta t$. The elastodynamic equations are integrated by a ADER-4 scheme [38, 24], which is explicit, fourth-order accurate in space and time, and stable when the CFL number is less than or equal to 1 .

The discretization of the interface requires special care to satisfy three criteria: (i) ensure a subcell resolution of the geometry; (ii) maintain the error of convergence despite the non-smoothness of the solution across the interface; (iii) discretize accurately the resonant jump conditions. For this purpose, we extend the Explicit Simplified Interface Method (ESIM) to the dissipative resonant case. Non-resonant case and inviscid resonant case have already been treated in [21] and [40], respectively.

The ESIM leads to distinguish two types of grid nodes [22]: the regular points for which the stencil of the ADER-4 scheme does not intersect the enlarged interface, and the irregular points for which the stencil includes at least one node whithin the enlarged interface where the solution is not defined. These grid nodes lying in the enlarged interface are called phantom points, where phantom values need to be built. A phantom value is a smooth extrapolation of the solution on the other side of the nearest interface. The next section describes how to compute the phantom values in 1D.

\subsection{Computation of the phantom values}

We denote by $(\boldsymbol{u})_{j}^{n}$ the numerical approximation of $\boldsymbol{u}$ at point $X_{j}=j \Delta X$ and time $t_{n}=n \Delta t$. $I_{L}$ and $I_{R}$ are the indices of the grid nodes that are the closest of the enlarged interface, see Figure 3. Due to the stencil of the ADER-4 scheme, there are four irregular points and four phantom points. The ESIM of order $q$ in 1D consists in defining the phantom values $\boldsymbol{u}_{*}\left(X_{j}, t_{n}\right)$ as the $q$-th order extrapolations of the solution at the grid node with index $j$ from the values of the solution at the physical point $\pm a / 2$, which may not coincide with a grid point. To do so, one introduces $\mathbb{T}_{j}^{q}( \pm a / 2)$ 

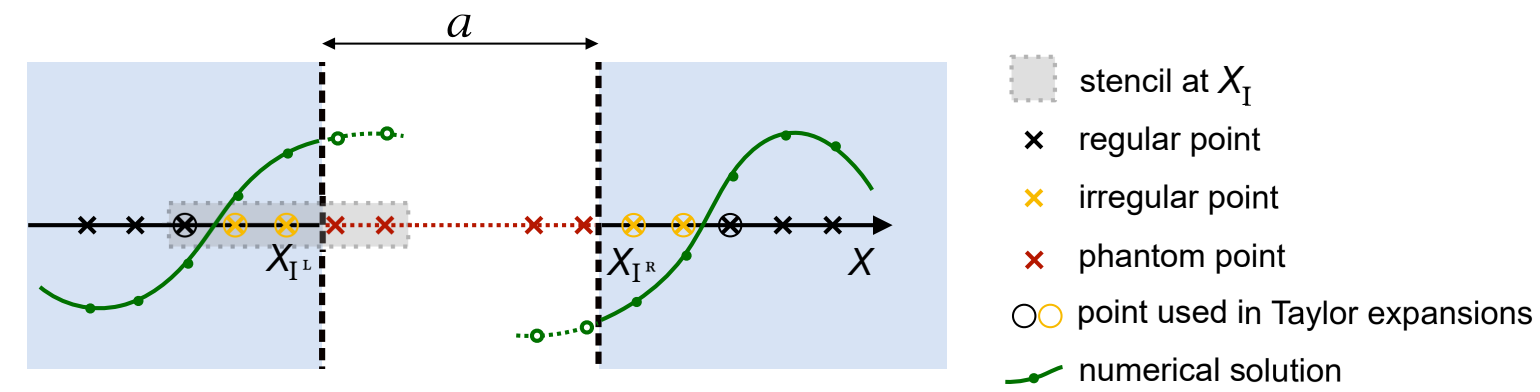

Figure 3: Stencil at $X_{I_{L}}$ and nodes around the enlarged interface.

as the $2 \times q_{U}$ matrices of the polynomial forms of the Taylor expansions:

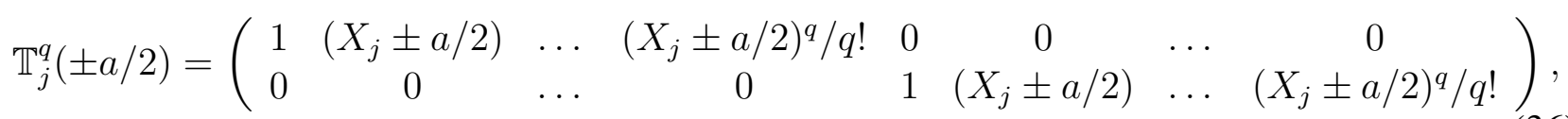

with $q_{U}=(2 q+2)$. The vectors $\boldsymbol{U}_{+}^{q}\left(t_{n}\right)$ and $\boldsymbol{U}_{-}^{q}\left(t_{n}\right)$ are also introduced and concatenate $q_{U}$ unknowns which are the traces on each side of the spatial derivatives of the fields $V$ and $\Sigma$ up to order $q$ :

$$
\boldsymbol{U}_{ \pm}^{q}\left(t_{n}\right)=\left(V_{ \pm}\left(t_{n}\right), \partial_{X} V_{ \pm}\left(t_{n}\right), \ldots, \partial_{X}^{q} V_{ \pm}\left(t_{n}\right), \Sigma_{ \pm}\left(t_{n}\right), \partial_{X} \Sigma_{ \pm}\left(t_{n}\right), \ldots, \partial_{X}^{q} \Sigma_{ \pm}\left(t_{n}\right)\right)^{\top} \in \mathbb{R}^{q_{U}},
$$

where $V_{ \pm}\left(t_{n}\right) \equiv V\left( \pm a / 2, t_{n}\right)$ and $\Sigma_{ \pm}\left(t_{n}\right) \equiv \Sigma\left( \pm a / 2, t_{n}\right)$ and the same notation is employed for the spatial derivatives. The numbering of arrays begin at 1: for instance, one has $\boldsymbol{U}_{ \pm}^{q}\left(t_{n}\right)[1]=$ $V\left( \pm a / 2, t_{n}\right)$. The phantom values in $1 \mathrm{D}$ can thus be defined:

$$
\begin{cases}\boldsymbol{u}_{*}\left(X_{j}, t_{n}\right)=\mathbb{T}_{j}^{q}\left(-\frac{a}{2}\right) \boldsymbol{U}_{-}^{q}\left(t_{n}\right) & \text { for } j=I_{L}+1, I_{L}+2, \\ \boldsymbol{u}_{*}\left(X_{j}, t_{n}\right)=\mathbb{T}_{j}^{q}\left(\frac{a}{2}\right) \boldsymbol{U}_{+}^{q}\left(t_{n}\right) & \text { for } j=I_{R}-1, I_{R}-2 .\end{cases}
$$

The method to derive the numerical approximations of the phantom values $\left(\boldsymbol{u}_{*}\right)_{j}^{n}$ is unchanged but the expression of the matrices is modified compared to the case $\gamma=+\infty$. The main steps are reminded in Appendix A and yield:

$$
\begin{cases}\left(\boldsymbol{u}_{*}\right)_{j}^{n}=\mathbb{T}_{j}^{q}\left(\boldsymbol{U}_{-}^{q}\right)^{n} & \text { for } j=I_{L}+1, I_{L}+2 \\ \left(\boldsymbol{u}_{*}\right)_{j}^{n}=\mathbb{T}_{j}^{q}\left(\boldsymbol{U}_{+}^{q}\right)^{n} & \text { for } j=I_{R}-2, I_{R}-1\end{cases}
$$

with

$$
\left\{\begin{array}{l}
\left(\boldsymbol{U}_{-}^{q}\right)^{n}=\left(\mathbb{M}^{q}\right)^{-1}\left[\mathcal{U}^{n}+\mathbb{F}^{q} \mathbb{Q}^{q} \boldsymbol{Z}^{n}\right] \\
\left(\boldsymbol{U}_{+}^{q}\right)^{n}=\left(\mathbb{C}_{+}^{q}\right)^{-1}\left[\mathbb{C}_{-}^{q}\left(\mathbb{M}^{q}\right)^{-1} \mathcal{U}^{n}+\mathbb{G}^{q} \mathbb{Q}^{q} \boldsymbol{Z}^{n}\right]
\end{array}\right.
$$

the numerical approximations of $\boldsymbol{U}_{ \pm}^{q}\left(t_{n}\right)$.

The matrices $\mathbb{T}_{j}^{q}\left( \pm \frac{a}{2}\right), \mathbb{C}_{ \pm}^{q}, \mathbb{Q}^{q},\left(\mathbb{M}^{q}\right), \mathbb{F}^{q}$, and $\mathbb{G}^{q}$ are defined in (36), (A.1), (A.6), (A.7). They are computed during a pre-processing step once for all. Their expressions are given in Appendix A for $q=3$. In practice, the computation of the phantom values given by (39) and (40) at each time step only requires to compute $\mathcal{U}^{n}$ and $\boldsymbol{Z}^{n} . \mathcal{U}^{n}$ is defined in (A.7) and is computed from the numerical values of the solution at the previous time. $Z^{n}$ is the numerical approximation of the vector containing the auxiliary variables defined in (A.2) and its computation is presented in the next section. 
For the sake of brevity, the numerical modeling in 2D is not detailed in this paper. It has been addressed in the inviscid case in [40]. The following two remarks highlight the main differences due to the incorporation of dissipation.

Remark 1. The computation of the phantom values in the two-dimensional inviscid case is detailed in [40]. Compared with the one-dimensional damped case of section (4.2), a significant change occurs when the jump conditions are derived q times, see (A.1). Indeed, the q-th order jump conditions are derived not only by differentiating in time the jump conditions but also by differentiating with respect to the curvilinear abscissa $s$. For the derivatives with respect to $s$, one uses $\partial_{s}=X_{1}^{\prime}(s) \partial_{X_{1}}+X_{2}^{\prime}(s) \partial_{X_{2}}$. Consequently, the $2 D$ version of the vector $Z^{n}$ is the vector of size $q_{A}=q(q+1) \times N_{R}$ that contains the auxiliary variables and their spatial derivatives until order $q-1$. Without dissipation, we only had $(q-2)(q-1) \times N_{R}$ auxiliary variables in $2 D$ since the second member only involves the third-order derivative term. In particular, the case $q=3$ used in practice did not require to introduce additional auxiliary variables in the inviscid case. Such technicalities can not be avoided with dissipation.

Remark 2. Without dissipation, the matrices in the ESIM separate the terms of stress and velocity, which have very different orders of magnitude. In the dissipative case, these terms are mixed, which induces a bad conditioning of the jump condition matrices and leads to numerical instabilities. To overcome this difficulty, normalized physical parameters are used in our simulation program when dissipation is considered. These quantities are denoted by tilds in the following. We choose a real $\mathcal{N}$ and define the normalized time $\tilde{t}=\mathcal{N} t$ and frequency $\tilde{f}=f / \mathcal{N}$ and the normalized physical parameters $\tilde{\rho}=\mathcal{N}^{3} \rho, \tilde{\mu}=\mathcal{N} \mu, \tilde{c}=c / \mathcal{N}, \tilde{\gamma}=\gamma / \mathcal{N}^{2}$.

\subsection{Computation of the auxiliary variables}

Compared with the inviscid case [40], the computation of the auxiliary variables is substantially changed by dissipation, since the equation satisfied by the auxiliary variables is modified. From now on the following assumption is used regarding the dissipation parameter $\gamma$ :

Assumption 2. $\gamma>\gamma^{\star}=1 /\left(2 \rho_{i} \omega_{1}\right)$.

The infinite set of resonant frequencies is strictly increasing (13), hence the assumption (2) implies $\gamma>1 /\left(2 \rho_{i} \omega_{r}\right)$ for all $r \geq 1$. The case of high -dissipation such that $\gamma \leq \gamma^{\star}$ yields a finite set of purely damped eigenmodes. The reader interested in this regime can easily adapt the forthcoming calculations. We introduce

$$
\left\{\begin{array}{l}
\xi^{1}=-1 /\left(2 \gamma \rho_{i}\right) \\
\xi_{r}^{2}=\sqrt{4 \omega_{r}^{2}-1 /\left(\gamma \rho_{i}\right)^{2}} / 2 \\
\varphi(\tau)=\mu_{m} \alpha_{r}^{2}\left(\partial_{t}+\frac{2}{\gamma \rho_{i}}\right)\left\langle\partial_{X}^{2} V\right\rangle_{a}(\tau)+\left(\frac{\alpha_{r}}{\gamma \rho_{i}}\right)^{2}\left\langle\partial_{X} \Sigma\right\rangle_{a}(\tau) .
\end{array}\right.
$$

Zero initial conditions are assumed for $J_{r}$ and $G_{r}$. Using Assumption 2, then the auxiliary fields write

$$
\left\{\begin{array}{l}
J_{r}\left(t_{n}\right)=\mathrm{e}^{\xi^{1} t_{n}}\left[-\Lambda_{r}^{1}\left(t_{n}\right) \cos \left(\xi_{r}^{2} t_{n}\right)+\Lambda_{r}^{2}\left(t_{n}\right) \sin \left(\xi_{r}^{2} t_{n}\right)\right], \\
G_{r}\left(t_{n}\right)=\mathrm{e}^{\xi^{1} t_{n}}\left[\Lambda_{r}^{1}\left(t_{n}\right)\left(\xi_{r}^{2} \sin \left(\xi_{r}^{2} t_{n}\right)-\xi^{1} \cos \left(\xi_{r}^{2} t_{n}\right)\right)+\Lambda_{r}^{2}\left(t_{n}\right)\left(\xi_{r}^{2} \cos \left(\xi_{r}^{2} t_{n}\right)+\xi^{1} \sin \left(\xi_{r}^{2} t_{n}\right)\right)\right],
\end{array}\right.
$$


where one has for $k=1,2$ :

$$
\Lambda_{r}^{k}\left(t_{n}\right)=\frac{1}{\xi_{r}^{2}} \int_{0}^{t_{n}} \ell_{r}^{k}(\tau) \mathrm{d} \tau \quad \text { with }\left\{\begin{array}{l}
\ell_{r}^{1}(\tau)=\varphi(\tau) \sin \left(\xi_{r}^{2} \tau\right) \mathrm{e}^{-\xi^{1} \tau} \\
\ell_{r}^{2}(\tau)=\varphi(\tau) \cos \left(\xi_{r}^{2} \tau\right) \mathrm{e}^{-\xi^{1} \tau}
\end{array}\right.
$$

This leads to an iterative computation of the functions $\Lambda_{r}^{k}$ :

$$
\left\{\begin{array}{l}
\Lambda_{r}^{k}\left(t_{0}\right)=0 \\
\Lambda_{r}^{k}\left(t_{n+1}\right)=\Lambda_{r}^{k}\left(t_{n}\right)+\frac{1}{\xi_{r}^{2}} \int_{t_{n}}^{t_{n+1}} \ell_{r}^{k}(\tau) \mathrm{d} \tau
\end{array}\right.
$$

where $t_{0}=0$. The integral is computed using an extrapolative Newton-Cotes formula [32]:

$$
\int_{t_{n}}^{t_{n+1}} \ell_{r}^{k}(\tau) \mathrm{d} \tau=\Delta t \sum_{w=0}^{1} \delta_{w} \ell_{r}^{k}\left(t_{n-w}\right)+\mathcal{O}\left(\Delta t^{3}\right)
$$

where $\delta_{0}=3 / 2$ and $\delta_{1}=-1 / 2$. The computation of the terms $\ell_{r}^{k}\left(t_{n-w}\right)$ requires to approximate $\varphi\left(t_{n-w}\right)$. The temporal derivative in (41) is computed using a finite-difference approximation:

$$
\partial_{t}\left\langle\partial_{X}^{2} V\right\rangle_{a}\left(t_{n-w}\right)=\frac{1}{\Delta t} \sum_{z=0}^{2} \beta_{z}\left\langle\partial_{X}^{2} V\right\rangle_{a}\left(t_{n-w-z}\right)+\mathcal{O}\left(\Delta t^{2}\right)
$$

with $\beta_{0}=3 / 2, \beta_{1}=-4 / 2$ and $\beta_{2}=1 / 2$. The numerical approximation of the traces at $t_{s} \leq t_{n}$ is computed thanks to the numerical approximations $\left(\boldsymbol{U}_{ \pm}^{q}\right)^{s}$ of (40):

$$
\left\{\begin{array}{l}
\left(\left\langle\partial_{X}^{2} V\right\rangle_{a}\right)^{s}=\frac{1}{2}\left(\left(\boldsymbol{U}_{-}^{q}\right)^{s}+\left(\boldsymbol{U}_{+}^{q}\right)^{s}\right)[3] \\
\left(\left\langle\partial_{X} \Sigma\right\rangle_{a}\right)^{s}=\frac{1}{2}\left(\left(\boldsymbol{U}_{-}^{q}\right)^{s}+\left(\boldsymbol{U}_{+}^{q}\right)^{s}\right)[q+3] .
\end{array}\right.
$$

To compute the numerical approximation $\left(\Lambda_{r}^{k}\right)^{n+1}$ of $\Lambda_{r}^{k}\left(t_{n+1}\right)$, the iterative relation (44) is used, the highest-order terms in (45) and (46) are neglected, and the approximation (47) is followed. It yields:

$$
\left\{\begin{array}{l}
\left(\Lambda_{r}^{k}\right)^{0}=0, \\
\left(\Lambda_{r}^{k}\right)^{n+1}=\left(\Lambda_{r}^{k}\right)^{n}+\frac{\alpha_{r}^{2} \Delta t}{\xi_{r}^{2}} \sum_{w=0}^{1} \delta_{w} \times \\
{\left[\frac{\mu_{m}}{\Delta t} \sum_{z=0}^{2} \beta_{z}\left(\left\langle\partial_{X}^{2} V\right\rangle_{a}\right)^{n-w-z}+\frac{2 \mu_{m}}{\gamma \rho_{i}}\left(\left\langle\partial_{X}^{2} V\right\rangle_{a}\right)^{n-w}+\frac{1}{\left(\gamma \rho_{i}\right)^{2}}\left(\left\langle\partial_{X} \Sigma\right\rangle_{a}\right)^{n-w}\right]\left(\kappa_{r}^{k}\right)^{n-w},}
\end{array}\right.
$$

with $\left(\left\langle\partial_{X}^{2} V\right\rangle_{a}\right)^{s}$ and $\left(\left\langle\partial_{X} \Sigma\right\rangle_{a}\right)^{s}$ given by (47), $\left(\kappa_{r}^{1}\right)^{s}=\sin \left(\xi_{r}^{2} t_{s}\right) \mathrm{e}^{-\xi^{1} t_{s}}$ and $\left(\kappa_{r}^{2}\right)^{s}=\cos \left(\xi_{r}^{2} t_{s}\right) \mathrm{e}^{-\xi^{1} t_{s}}$. Then, the numerical approximation of the auxiliary variables writes:

$$
\left\{\begin{array}{l}
J_{r}^{n}=\mathrm{e}^{\xi^{1} t_{n}}\left[-\left(\Lambda_{r}^{1}\right)^{n} \cos \left(\xi_{r}^{2} t_{n}\right)+\left(\Lambda_{r}^{2}\right)^{n} \sin \left(\xi_{r}^{2} t_{n}\right)\right] \\
G_{r}^{n}=\mathrm{e}^{\xi^{1} t_{n}}\left[\left(\Lambda_{r}^{1}\right)^{n}\left(\xi_{r}^{2} \sin \left(\xi_{r}^{2} t_{n}\right)-\xi^{1} \cos \left(\xi_{r}^{2} t_{n}\right)\right)+\left(\Lambda_{r}^{2}\right)^{n}\left(\xi_{r}^{2} \cos \left(\xi_{r}^{2} t_{n}\right)+\xi^{1} \sin \left(\xi_{r}^{2} t_{n}\right)\right)\right] .
\end{array}\right.
$$

It follows the vector $\boldsymbol{Z}^{n}$ and consequently the phantom values (39). 
The numerical method presented in sections 4.2-4.3 involves different parameters: order $K=4$ of the ADER schema, order $q=3$ of the ESIM, order $q_{I}=3$ of the integration (45), and order $q_{D}=2$ of the derivation (46). In the inviscid case [40], the local truncation error was analysed. The steps of the proof can be adapted directly to the dissipative case, and are not repeated here. We then have the following result.

Property 1. Let assume that the numerical method is stable. Then the total error $\varepsilon\left(t_{n}\right)=\mathcal{O}\left(\Delta X^{\nu}\right)$ is obtained, with the parameter

$$
\nu=\min \left(K, q-1, q_{I}-1, q_{D}\right), \quad \text { for } q=\{3,5\}
$$

In the present case, it follows convergence of order $\nu=2$. This theoretical insight will be verified numerically in section 5 .

\section{Numerical experiments}

In Section 5.1, we first detail the microstructured configuration under study. Section 5.2 focuses on the validation of the numerical method: comparisons with analytical solutions and convergence measurements validate the numerical methods in both 1D and 2D. Then, Section 5.3 compares the simulation results with microstructured and homogenized configuration. Different frequencies are considered, illustrating the accuracy of the homogenization method. Two types of sources are considered; the source point case allows to impact the microstructures with different angles, which solicits all terms in the homogenized case. Finally, Section 5.4 proposes a physical analysis of the results, in a 1D geometry. The analysis of the scattering matrix allows to find a critical value of attenuation and a frequency for which the waves impacting the interface are totally absorbed. Time-domain simulations illustrate that this phenomenon is very fastly reached.

\subsection{Microstructured and homogenized configurations}

We consider a straight array of elliptic inclusions defined by their semi-major axis $r_{1}=0.8 \mathrm{~m}$, semi-minor axis $r_{2}=0.5 \mathrm{~m}$ and tilt angle $\theta=40^{\circ}$ with respect to the $X_{1}$-axis. This results in an array of thickness $e=1.3838 \mathrm{~m}$, with a periodicity $h=2 \mathrm{~m}$. The constitutive moduli are $\mu_{m}=10^{10} \mathrm{~kg} \cdot \mathrm{m}^{-1} \cdot \mathrm{s}^{-2}, \mu_{i}=10^{-2} \mu_{m}=10^{8} \mathrm{~kg} \cdot \mathrm{m}^{-1} \cdot \mathrm{s}^{-2}$, and $\rho_{i}=\rho_{m}=4.44 \cdot 10^{3} \mathrm{~kg} \cdot \mathrm{m}^{-3}$ with a high contrast in the shear modulus and no constrast in the density. The resulting velocities are $c_{m}=1500 \mathrm{~m} \cdot \mathrm{s}^{-1}$ and $c_{i}=150 \mathrm{~m} \cdot \mathrm{s}^{-1}$. The minimal dissipation parameter in Assumption 2 is $\gamma^{\star}=1.8710^{-7} \mathrm{~kg}^{-1} \cdot \mathrm{m}^{3} \cdot \mathrm{s}$. The dissipation parameter $\gamma$ is given for each numerical experiment later on.

The numerical approximation in this microstructured configuration is computed using the numerical method presented in [23]: the system is discretized using the ADER-4 scheme and the geometry of the inclusions is handled using the ESIM. It yields high numerical costs because the mesh size has to be much smaller than the caracteristic size of the inclusions. The dissipation in the inclusion is taken into account with a Strang's splitting method [18]. The value of the normalization parameter is set to $\mathcal{N}=10^{3}$.

The corresponding homogenized model is given by (34). Due to the energy considerations mentionned in Section 3, the thickness of the enlarged interface is chosen such that $a=e$. The effective parameters used in these jump conditions are given in Tables 1 and 2. The numerical 


$$
\begin{array}{|c|c|c|c|c|}
\widetilde{B}_{1} & \widetilde{B}_{2} & \widetilde{C}_{11} & \widetilde{C}_{12} & \widetilde{C}_{22} \\
\hline 3.206 & -0.284 & 0.756 & -0.284 & 0.4
\end{array}
$$

Table 1: Numerical values of the effective interface parameters featured in (34).

approximation of the homogenized solution is obtained using the numerical method presented in Section 4.

For both configurations, we will consider either initial conditions for $\boldsymbol{u}=\left(V, \Sigma_{1}, \Sigma_{2}\right)^{\top}$ or a source point. The initial conditions read

$$
\boldsymbol{u}(\boldsymbol{X}, 0)=\left(\begin{array}{c}
\frac{1}{\mu_{m}} \\
-\frac{1}{c_{m}} \\
0
\end{array}\right) G\left(\left(\boldsymbol{X}-\boldsymbol{X}_{I}\right) \cdot \boldsymbol{e}_{1}\right),
$$

where $\boldsymbol{X}_{I}$ is an initialization point chosen to control the compact support of the initial condition. The source point is located at $\boldsymbol{X}_{S}$ such that (1) reads:

$$
\left\{\begin{array}{l}
\frac{\partial \boldsymbol{\Sigma}}{\partial t}(\boldsymbol{X}, t)=\mu \boldsymbol{\nabla} V(\boldsymbol{X}, t) \\
\rho \frac{\partial V}{\partial t}(\boldsymbol{X}, t)+\frac{1}{\gamma} V(\boldsymbol{X}, t)=\operatorname{div} \boldsymbol{\Sigma}(\boldsymbol{X}, t)+\rho \delta\left(\boldsymbol{X}-\boldsymbol{X}_{s}\right) G\left(t c_{m}\right),
\end{array}\right.
$$

with $\delta\left(\boldsymbol{X}-\boldsymbol{X}_{s}\right)$ being a Dirac delta function at $\boldsymbol{X}=\boldsymbol{X}_{s}$. The source function $G$ used in both cases is defined by:

$$
G(x)= \begin{cases}\sum_{k=0}^{3} \alpha_{k} \sin \left(2^{k} k_{m} x\right) & \text { if }-\frac{c_{m}}{f_{0}} \leq x \leq 0 \\ 0 & \text { otherwise }\end{cases}
$$

with $f_{0}$ the central frequency, $\alpha_{0}=1, \alpha_{1}=-21 / 32, \alpha_{2}=63 / 768$, and $\alpha_{3}=-1 / 512$, such that $G$ is of class $C^{6}$. The typical wavelength in the matrix is defined as $\lambda_{0}=\frac{c_{m}}{f_{0}}$, so that the associated small parameter of Assumption 1 is

$$
\eta\left(f_{0}\right)=\frac{2 \pi f_{0} h}{c_{m}} .
$$

\subsection{Validation of the numerical method}

\subsubsection{ID setting}

We consider a microstructured array aligned with the $X_{2}$-axis and centered at $X_{1}=0$ together with the initial conditions (50). Consequently, in the homogenized problem, the incident plane wave impacts the enlarged at normal incidence. Thus, the homogenized configuration can be considered as

\begin{tabular}{|c|c|c|c|c|c|c|c|c|c|}
\hline$r$ & 0 & 1 & 4 & 8 & 9 & 13 & 16 & 21 & 23 \\
\hline$\alpha_{r}$ & 0.314 & 0.462 & 0.144 & 0.148 & 0.069 & 0.078 & 0.037 & 0.053 & 0.081 \\
\hline$\omega_{r}\left(\mathrm{rad} \cdot \mathrm{s}^{-1}\right)$ & & 600 & 1101 & 1523 & 1637 & 1962 & 2178 & 2438 & 2463 \\
\hline
\end{tabular}

Table 2: Resonances parameters in (23). When $\alpha_{r}=0$, the value of $\omega_{r}$ is not given. 
a one-dimensional problem. In this case, the numerical method has been developped in Sections 4.1, 4.2 and 4.3. The present section aims at validating this approach to simulate the wave propagation in the effective medium.

The central frequency is $f_{0}=72 \mathrm{~Hz}$ in the source function (52) used in the initial conditions (50). The point $\boldsymbol{X}_{I}=(-2,0) \mathrm{m}$ is chosen such that the initial conditions do not intersect the enlarged interface. The initial profile (normalized by its maximum) of the velocity is displayed in Figure 4a. The numerical parameters are $\Delta X=0.2 \mathrm{~m}$ for the mesh size and $\Delta t=\zeta \Delta X / c_{m}$ for the time step. The CFL number $\zeta=0.95<1$ is chosen and ensures the stability of the ADER-4 scheme in a homogeneous medium. The order of the ESIM is $q=3$.

The dissipative parameter is $\gamma=2 \cdot 10^{-6} \mathrm{~kg}^{-1} \cdot \mathrm{m}^{3} \cdot \mathrm{s}>\gamma^{\star}$. The profile of the numerical approximation of this homogenized model is displayed at the final time $t_{f}=31.7 \mathrm{~ms}$ together with a semi-analytical solution $V_{\text {ref }}$ in Figure $4 \mathrm{~b}$. The computation of this semi-analytical solution is detailed in Section 3.2.

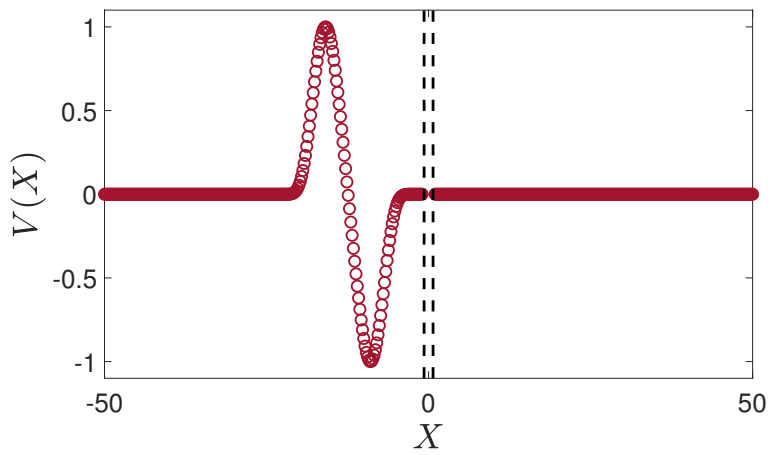

(a) $t=0$

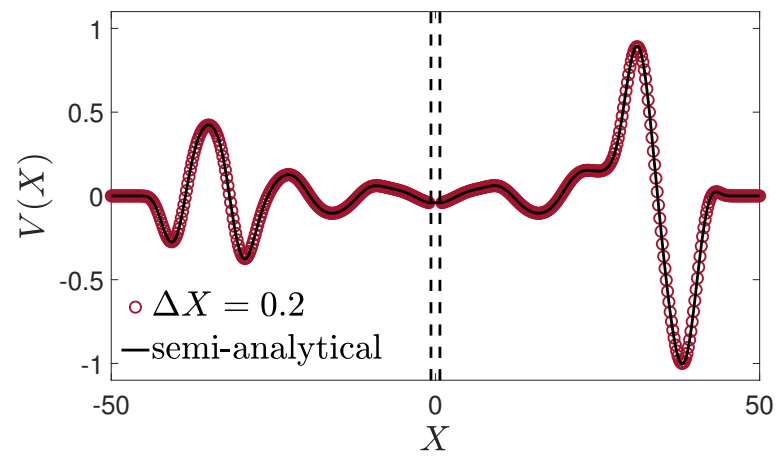

(b) $t=t_{f}$

Figure 4: Velocity profiles of the homogenized problem at time $t=0$ and time $t=t_{f}$ for a plane wave at normal incidence with $f_{0}=72 \mathrm{~Hz}$. The dashed lines denote the positions of the enlarged interface.

The discrepancy between the numerical and semi-analytical homogenized solutions is measured in the $L^{2}$-norm for $X \in[-50 ;-5] \mathrm{m}$. The relative error in Figure $4 \mathrm{~b}$, i.e. for $\Delta X=0.2 \mathrm{~m}$, is of $0.7 \%$. The relative error is then represented in Figure 5 as a function of $\Delta X$ whose slope in a $\log$-log scale graph characterizes the global order of the scheme. This figure is obtained with the relative position of the enlarged interface in the uniform Cartesian grid being kept while the mesh size $\Delta X$ increases. The errors obtained in the case $\gamma=+\infty$ are also included in the figure for comparison. As theoretically predicted in Property 1, order 2 of convergence is measured. 


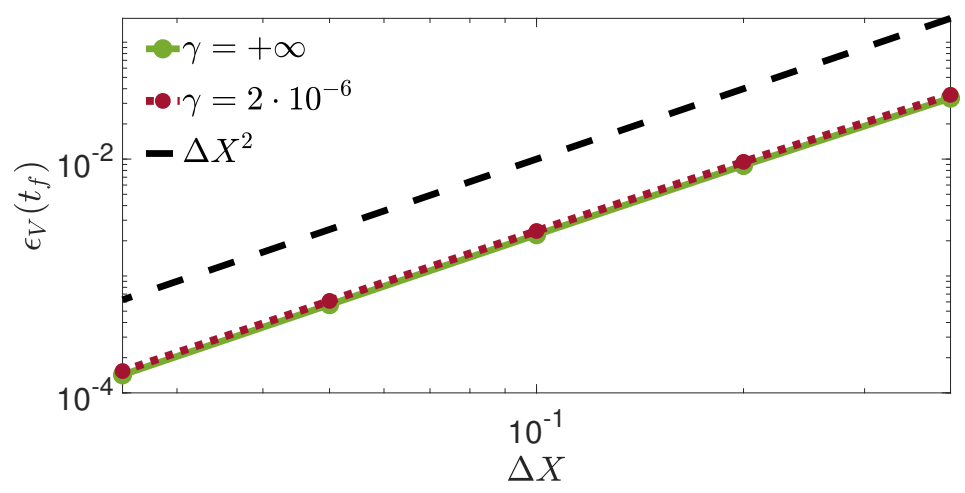

Figure 5: Relative error between the 1D numerical and semi-analytical velocities of the homogenized problem in a log-log scale.

\subsection{2. $2 D$ setting}

In this section, we validate the 2D numerical method mentioned in Remarks 1 and 2. We still consider the same configuration of an incident plane wave at normal incidence than in Section 5.2.1 but the full $2 \mathrm{D}$ algorithm is used for the homogenized problem. The same numerical parameters than in 1D are used, $q=3$ and $\Delta X=0.2 \mathrm{~m}$. Periodic conditions are imposed at the top and the bottom of the computational domain. For $f_{0}=72 \mathrm{~Hz}$, Figure 6 shows the velocity fields at the initial time $t=0$ and the final time $t=t_{f}=31.7 \mathrm{~ms}$. The profiles along $X_{2}=-1 \mathrm{~m}$ of the numerical and semi-analytical solutions of the homogenized problem are displayed at $t_{f}$ in Figure (7a). The discrepancy is of same order as in $1 \mathrm{D}$, with a relative error of $1 \%$. This relative error is displayed as a function of $\Delta X$ in Figure (7b) and confirms an order of 2. Consequently, we observe the same order of convergence as in $1 \mathrm{D}$ even if the $2 \mathrm{D}$ numerical method is much more intricate, involving 12 auxiliary variables computed instead of 2 for the one-dimensional problem, see Remark 1.

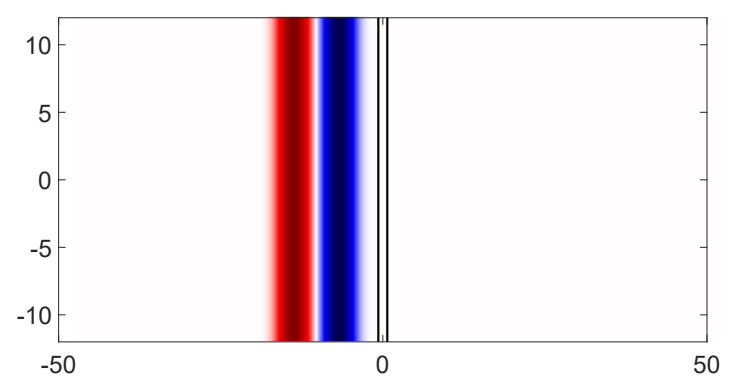

(a) $t=0$

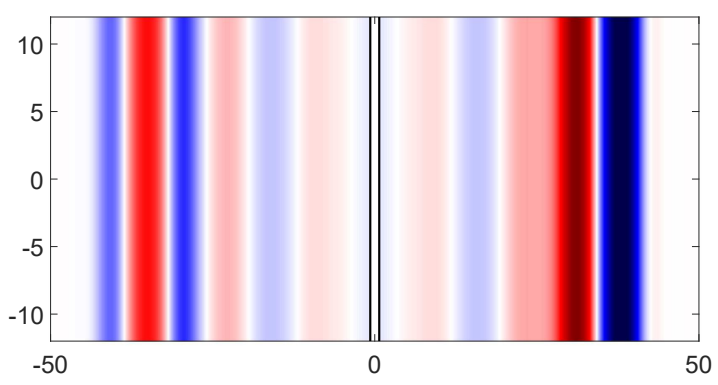

(b) $t=t_{f}$

Figure 6: Velocity fields of the homogenized problem at time $t=0$ and time $t=t_{f}$ at normal incidence with $f_{0}=72$ Hz. The black lines denote the enlarged interface.

\subsection{Validation of the homogenized model}

Once the numerical method validated, for a mesh size enough small, we compare the numerical simulations in the microstructured configuration and for the homogenized problem to assess the reliability of the jump conditions (34). 


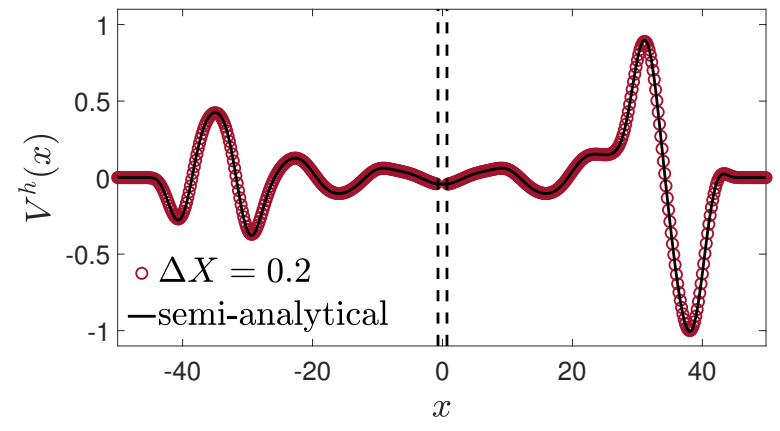

(a) Homogenized velocity profiles

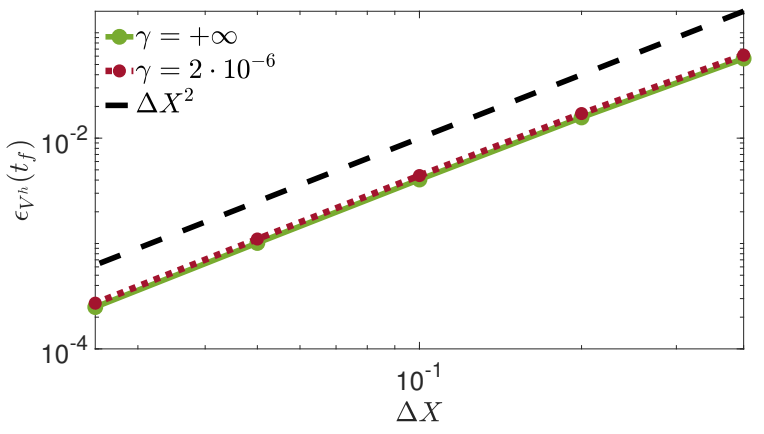

(b) Relative errors

Figure 7: Numerical and semi-analytical velocity profiles (a) of the homogenized problem at time $t=t_{f}$ for normal incidence with $f_{0}=72 \mathrm{~Hz}$ and corresponding relative errors (b).

\subsubsection{Incident plane wave at normal incidence}

We compare the homogenized simulations with full-field simulations performed in the microstructured configuration. The mesh size of $\Delta X=0.2 \mathrm{~m}$, which gives satisfying results according to Figure 7, is chosen for the homogenized configuration. On the other hand, a mesh size 8 times smaller $\Delta X=0.025 \mathrm{~m}$ is required to handle the geometry of the microstructure. This underlines the benefits of the homogenization process in terms of computational time and memory requirement. The dissipation parameter is $\gamma=2 \cdot 10^{-6} \mathrm{~kg}^{-1} \cdot \mathrm{m}^{3} \cdot \mathrm{s}$. One investigates the agreement between both configurations for three values of $\eta\left(f_{0}\right)=0.3,0.6,0.9$ in (53). This corresponds to central frequencies of $f_{0}=36,72,108 \mathrm{~Hz}$, respectively in (52) for the initial conditions (50).
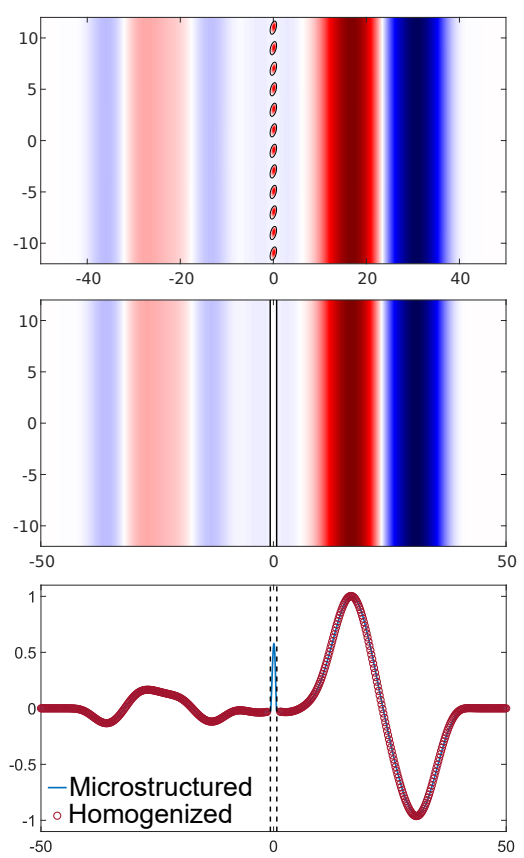

$\eta\left(f_{0}\right)=0.3$
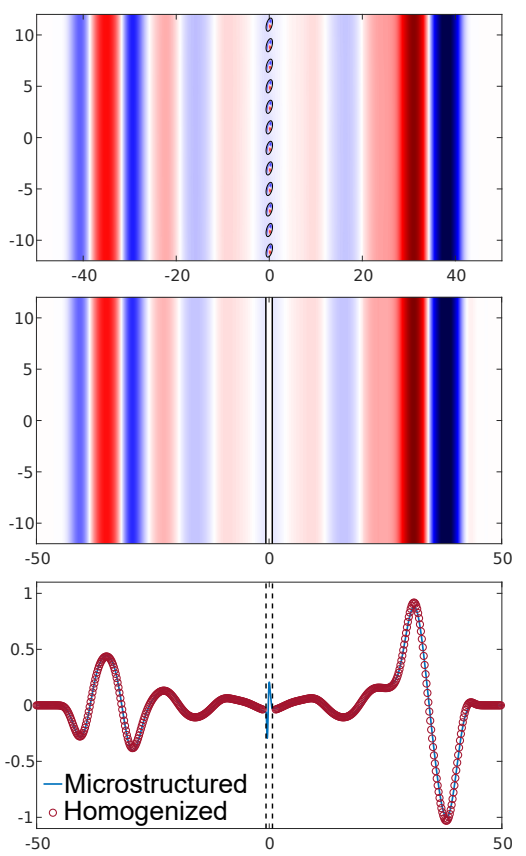

$\eta\left(f_{0}\right)=0.6$
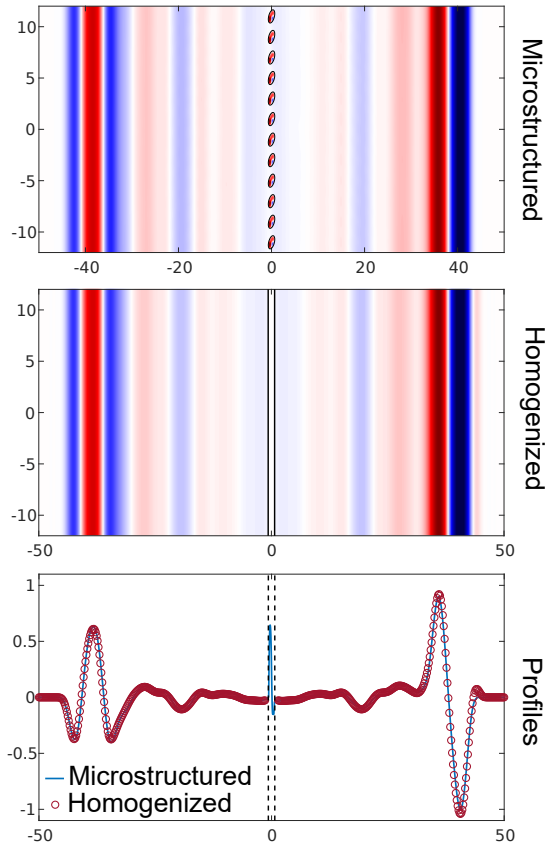

$\eta\left(f_{0}\right)=0.9$

Figure 8: (Top two rows) velocity fields for the microstructured configuration and for the homogenized model for an illumination by an incident plane wave at normal incidence. (Bottom row) comparison of the corresponding velocity profiles at $X_{2}=-1 \mathrm{~m}$. (left) $\eta\left(f_{0}\right)=0.3$, (center) $\eta\left(f_{0}\right)=0.6$, and (right) $\eta\left(f_{0}\right)=0.9$. 
The velocity fields for the microstructured configuration and for the homogenized model together with their respective profiles along $X_{2}=-1 \mathrm{~m}$ are reported in Figure 8 at time $t=31.7 \mathrm{~ms}$. The corresponding discrepancy between both solutions is measured in the $L^{2}$-norm for $X_{1} \in[-50 ;-5] \mathrm{m}$ and at $X_{2}=-1 \mathrm{~m}$. The relative errors are of about $3.8 \%, 5.7 \%$ and $7.4 \%$, respectively. As expected, the solutions agree at low frequency but deviate from one another as the parameter $\eta\left(f_{0}\right)$ increases to 1 and does not conform to Assumption 1. The Fourier spectrum of the source function $G$ is displayed as a function of $\eta(f)$ in Figure 9 for the three values of $\eta\left(f_{0}\right)$ used in the previous numerical experiments. One notes that this wide-band signal involves higher frequencies than $f_{0}$ for which the associated values $\eta(f)$ lie beyond the hypothesis of the small parameter of Assumption 1. The comparison of the simulations in both configurations is then satisfying given the large values of $\eta(f)$ involved.

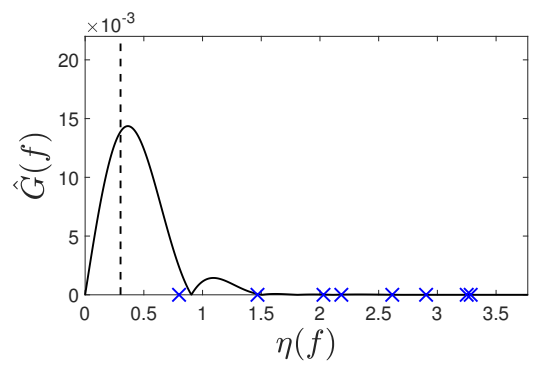

(a) $\eta\left(f_{0}\right)=0.3$

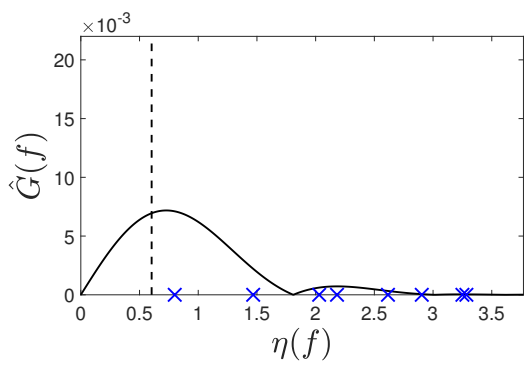

(b) $\eta\left(f_{0}\right)=0.6$

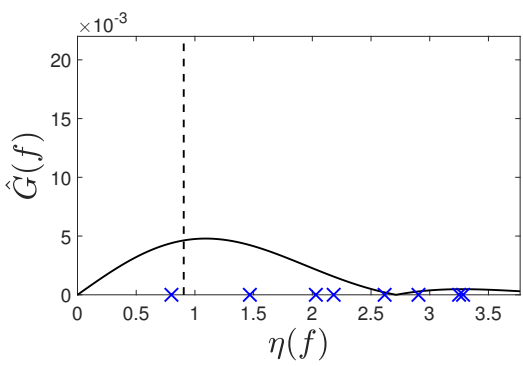

(c) $\eta\left(f_{0}\right)=0.9$

Figure 9: Fourier transform $\hat{G}$ of the source function for the three different values $\eta\left(f_{0}\right)$ considered (here indicated by the dashed lines). The blue crosses denote the values $\eta\left(\omega_{r} /(2 \pi)\right)=\omega_{r} h / c_{m}$ associated with resonances $\omega_{r}$ in (23).

In order to have a more precise comparison frequency by frequency, the transmission coefficients are now studied. To do so, the velocity of the microstructured configuration and the homogenized model in the case $\eta\left(f_{0}\right)=0.6$ is recorded from $t=0$ to $t=475 \mathrm{~ms}$ at one receiver located at $(10,0) \mathrm{m}$. The Fourier transform of the velocity is computed from these data and normalized by the one of the source function. The resulting transmission coefficient is displayed in Figure 10. One recovers the fact that the agreement between both transmission coefficients deteriorates around the resonant frequencies that are not taken into account in $\mathscr{D}_{\gamma}(\omega)$. Moreover, in the dissipative case $\gamma \neq \infty$, the behaviour of the transmission coefficient around the resonances is less singular than when damping is not considered. Consequently, around these resonances the agreement is better than the ones presented in [41] explaining the good quality of the results in Figure 8 even for the higher values of $\eta\left(f_{0}\right)$.

\subsubsection{Source point}

In the previous example, we had a $X_{2}$-invariance in the homogenized problem with consequently no role of the effective parameters $\widetilde{B}_{2}, \widetilde{C}_{12}$ and $\widetilde{C}_{22}$ in (34). In this section, we consider the illumination by a source point (51) located at $\boldsymbol{X}_{S}=(-35,0) \mathrm{m}$ so that all the effective parameters are taken into account. The numerical and physical parameters are unchanged and the computational domain is defined as $[-70 \mathrm{~m} ; 35 \mathrm{~m}] \times[-132 \mathrm{~m} ; 132 \mathrm{~m}]$. Perfectly Matched Layers are used in the background domain on the left and right boundaries while the domain is chosen large enough along the $X_{2}$-axis to avoid reflections from the top and bottom boundaries. The simulations of the homogenized model are compared with full-field simulations to investigate the capability of the homogenized problem to model a full 2D configuration. 


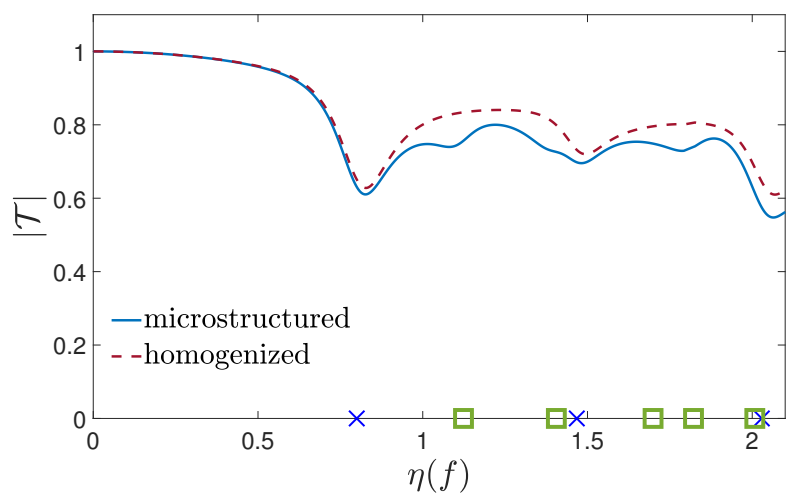

Figure 10: Transmission coefficient for the microstructured configuration and the homogenized model. The blue crosses and the green squares denote the resonant frequencies taken into account in $\mathscr{D}_{\gamma}(\omega)$ and the missed resonant frequencies of zero mean modes, respectively.

The velocity fields are displayed at times $t \in\left\{t_{1}, t_{2}, t_{3}, t_{4}\right\}=\{25.3,38.0,50.7,63.3\} \mathrm{ms}$ on the figures 11,12 and 13 for $\eta\left(f_{0}\right)=0.3,0.6,0.9$, respectively. Only a subset of the computational domain $[-62 \mathrm{~m} ; 27 \mathrm{~m}] \times[-40 \mathrm{~m} ; 40 \mathrm{~m}]$ is shown. The profiles at $X_{2}=1 \mathrm{~m}$ are then compared on Figure 14. Quantitatively, the discrepancies between solutions of the microstructured problem and of the homogenized model are measured in the $L^{2}$-norm for $X_{1} \in[-50 \mathrm{~m} ;-5 \mathrm{~m}]$ and at $X_{2}=1 \mathrm{~m}$. The relative errors for the three values of $\eta\left(f_{0}\right)$ are of about $2.7 \%, 7 \%$ and $14 \%$, respectively. A good agreement is again observed at low frequency and decreases as $\eta\left(f_{0}\right)$ increases to 1 .

\subsection{Application to Coherent Perfect Absorption}

In the previous examples, we validated the numerical methods and showed that the homogenization of interfaces represented very accurately resonant and dissipative microstructures. Consequently, we will now use numerical simulations of the homogenized configuration to investigate the effect of the dissipation parameter $\gamma$. For this purpose we will consider a plane wave at normal incidence, so that the problem becomes one-dimensional again. Numerical simulations are performed with $\Delta X=0.2 \mathrm{~m}$, which guarantees a good accuracy according to Figures 4 and 5 .

First, the velocity profiles at $t=t_{f}$ are presented in Figure 15 for different values of $\gamma$. The resonant behaviour of the material leads to energy radiated by the enlarged interface even a long time after the incident wave has passed it. Close to the limit value $\gamma^{\star}$, for example $\gamma=2 \cdot 10^{-7} \mathrm{~s} \cdot \mathrm{kg}^{-1} \cdot \mathrm{m}^{3}$, the dissipation is so high that we do not see this resonant behaviour anymore. On the other hand, close to $100 \gamma^{\star}$, for example $\gamma=2 \cdot 10^{-5} \mathrm{~kg}^{-1} \cdot \mathrm{m}^{3} \cdot \mathrm{s}$, the dissipation has barely any influence and we almost recover the case $\gamma=+\infty$.

Considering a homogenized interface is obviously very advantageous on a computational level. But it also makes it much easier to study the physical properties of the studied system. We then will illustrate this aspect, by focusing on the phenomenon of Coherent Perfect Absorption. Schematically, resonant and dissipative scatterers behave as open systems, characterized by their energy leakage and intrinsic losses. The equilibrium between these two energy losses corresponds to a condition of critical coupling and maximizes the energy absorption. The study of critical coupling is an active research topic in acoustics, especially for the design of subwavelength panels made of Helmholtz resonators, ie resonances induced by the geometry $[35,13,14,15,17,33,34]$. Here we propose an example with material resonances.

The setting is still one-dimensional: the design of an optimal metasurface over a wide band of 

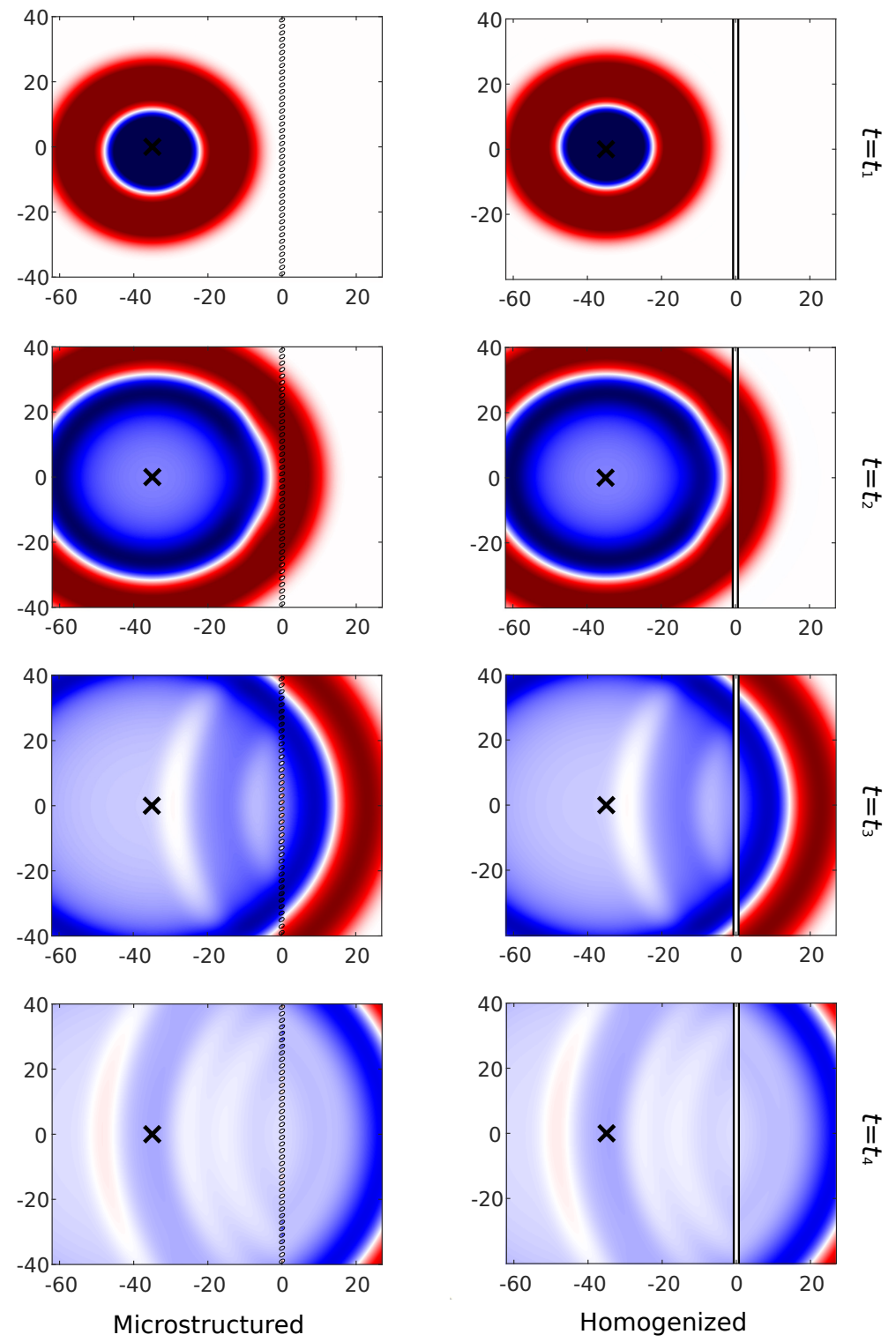

Figure 11: (Left) velocity fields for the microstructured configuration and (right) for the homogenized model for $\eta\left(f_{0}\right)=0.3$ and at different times $t \in\{25.3,38.0,50.7,63.3\} \mathrm{ms}$. A source point is located at the black cross.

incidence angles [14] is out of the scope of this paper. From the reflection and transmission coefficients for $\theta=0$ (25), one can fill in the scattering matrix $\mathbb{S}[34]$. The poles of $\operatorname{det}(\mathbb{S})$ are off the real axis, and have a real part close to each resonant frequency $\omega_{r}$ in $\mathscr{D}_{\gamma}(\omega)$. By convention, we have chosen a time dependence $e^{i \omega t}$, so that the poles are in the upper half-plane of $\mathbb{C}$, unlike [34]. For a medium without dissipation, the zeros of $\operatorname{det}(\mathbb{S})$ and complex conjugate of the poles, and lie therefore in the lower half-plane of $\mathbb{C}$. This property is observed in Figure $(16 a)$, where we represent $\log (|\operatorname{det}(\mathbb{S})|)$ around the first four resonance frequencies $\omega_{r}$ in $\mathscr{D}_{\gamma}(\omega)$.

When attenuation is considered in the scatterers, the position of these zeros and poles evolves in the complex plane. The imaginary parts of these quantities grow strictly with the magnitude of the dissipative effects. Associated to each resonance $\omega_{r}$, there is then a critical value of the attenuation for which the zero of $\operatorname{det}(\mathbb{S})$ crosses the real axis, at a frequency $\tilde{\omega}_{r}$. Figure (16b) illustrates this property; for the critical value $\gamma=4.47 \cdot 10^{-6}$, we observe that the zero of $\operatorname{det}(\mathbb{S})$ associated to the 

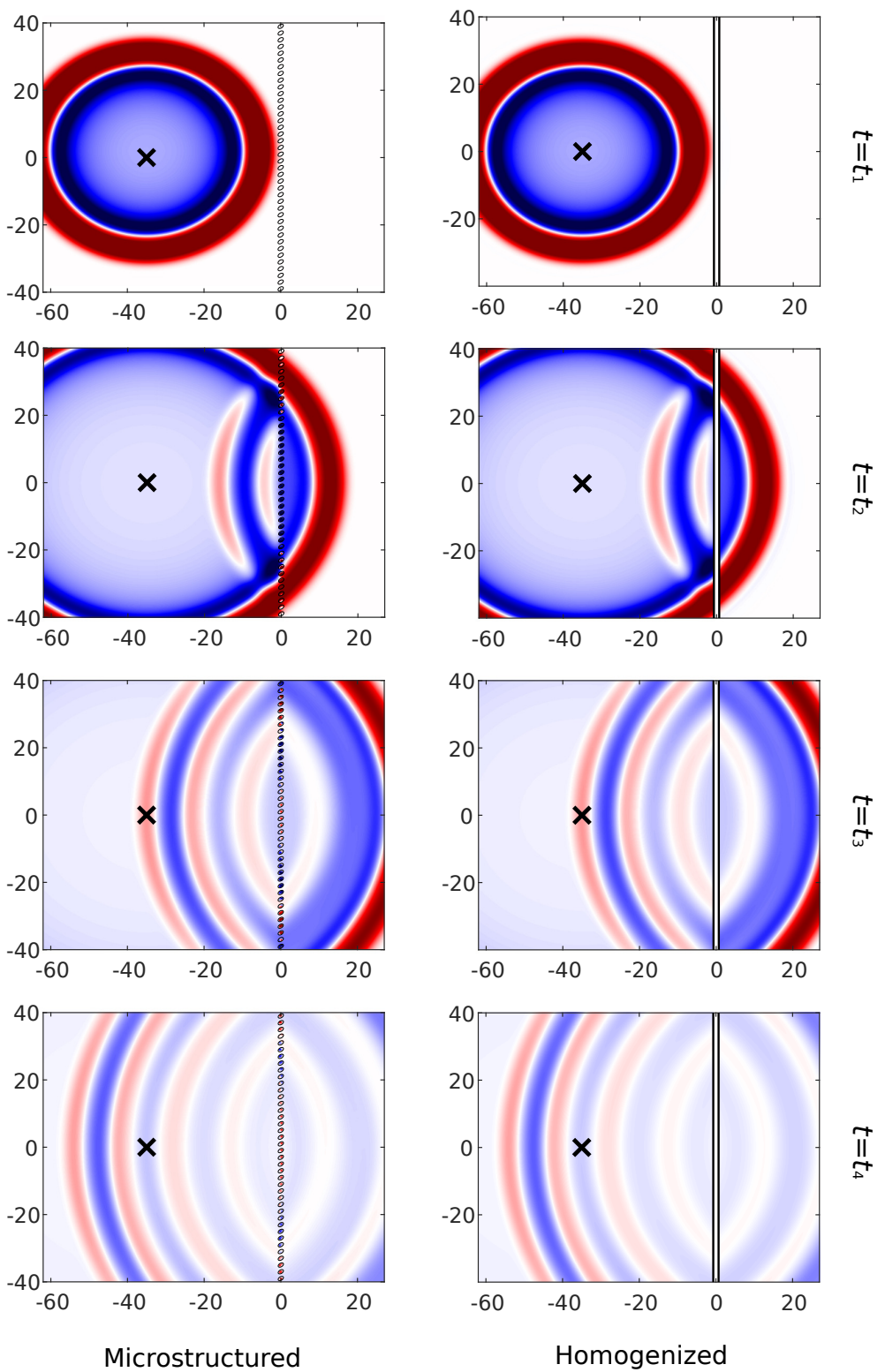

Figure 12: (Left) velocity fields for the microstructured configuration and (right) for the homogenized model for $\eta\left(f_{0}\right)=0.6$ and at different times $t \in\{25.3,38.0,50.7,63.3\} \mathrm{ms}$. A source point is located at the black cross.

first resonance frequency crosses the real axis. This is not the case for the three following resonances at this value of $\gamma$. Figure 17 displays the trajectory of the zeros of $\operatorname{det}(\mathbb{S})$ in the complex plane. We note that there is a slight shift between $\omega_{r}$ and $\tilde{\omega}_{r}$.

The transmission and reflexion coefficients computed analytically in (25) are displayed in Figure 18 for the two values $\gamma=+\infty$ and $\gamma=4.47 \cdot 10^{-6} \mathrm{~kg}^{-1} \cdot \mathrm{m}^{3} \cdot \mathrm{s}$; as said previously, this last value ensures the critical coupling at $\tilde{\omega}_{1}$. When dissipation is not considered, perfect transmission is reached followed by perfect reflection within a small band width around the resonant frequencies. We introduce $\omega_{\min , 1}$ the frequency for which transmission is minimal around the first resonance. With dissipation, these behaviors are smoothed. Besides, one notices that dissipation $\alpha$ is maximal around these resonant frequencies (see Figure 18c): as usual for transmission problems illuminated only on one side [34], the optimal value is $\alpha=0.5$ and it is reached at $\tilde{\omega}_{1}$. 

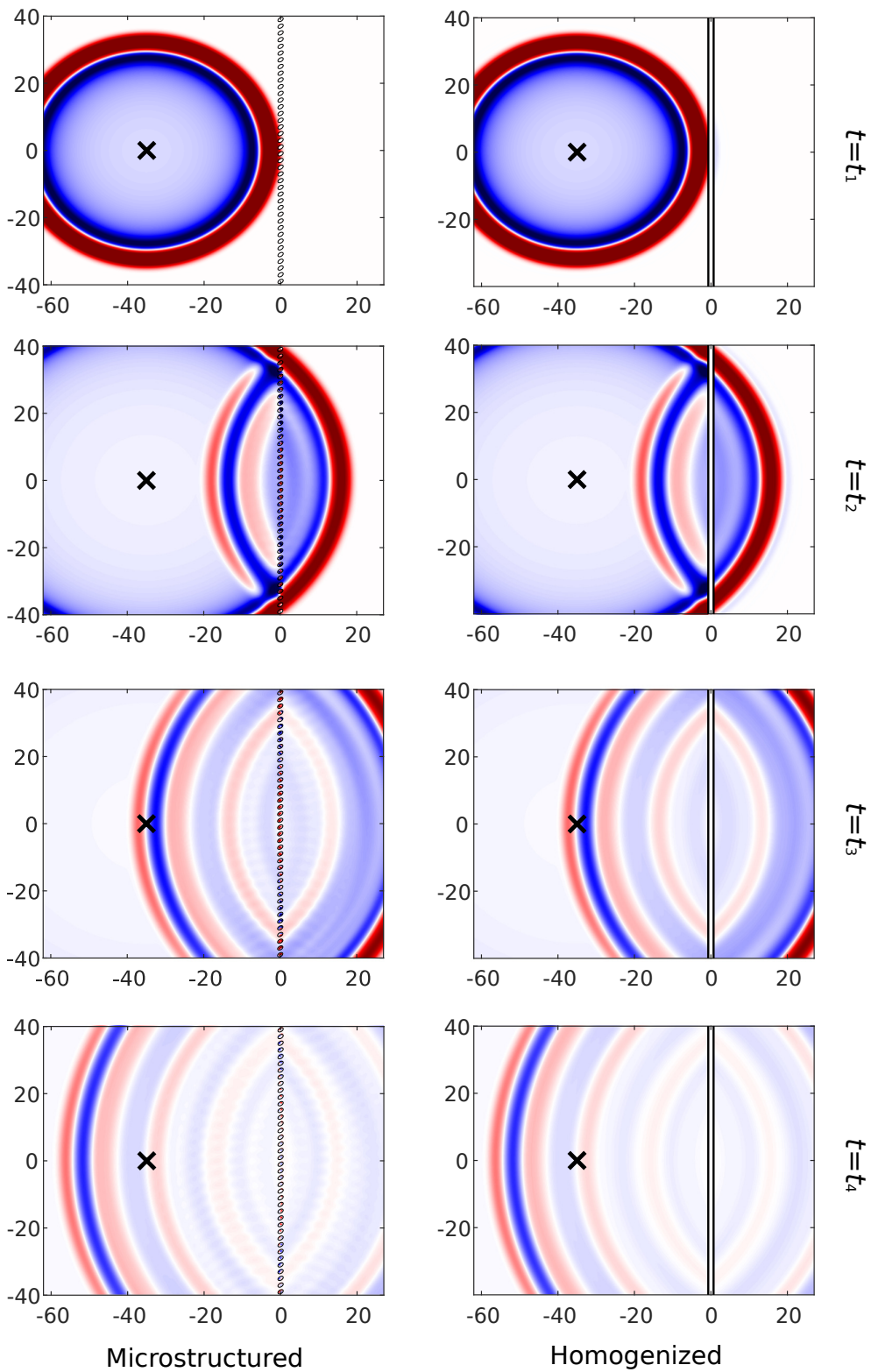

Figure 13: (Left) velocity fields for the microstructured configuration and (right) for the homogenized model for $\eta\left(f_{0}\right)=0.9$ and at different times $t \in\{25.3,38.0,50.7,63.3\} \mathrm{ms}$. A source point is located at the black cross.
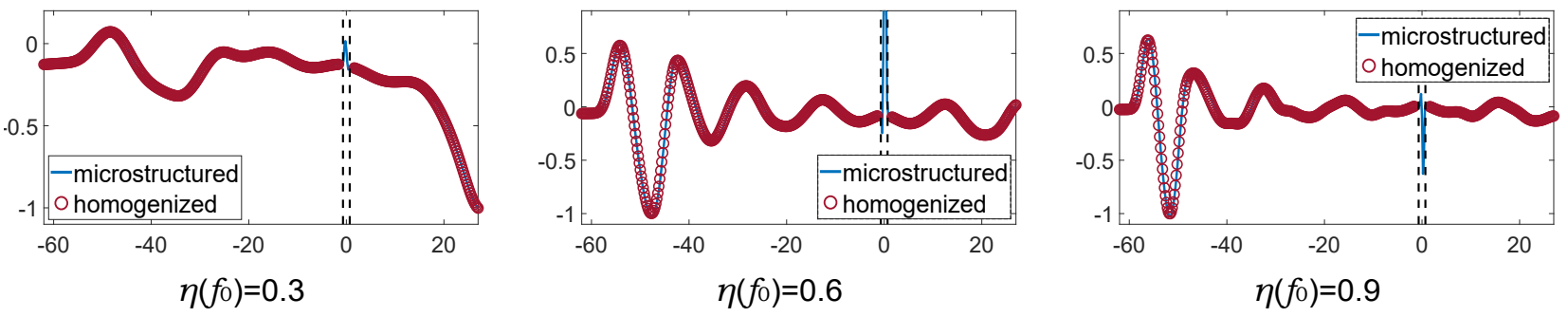

Figure 14: comparison of the velocity profiles at $X_{2}=1 \mathrm{~m}$ for the case of a source point and the three different values of $\eta\left(f_{0}\right)$. 


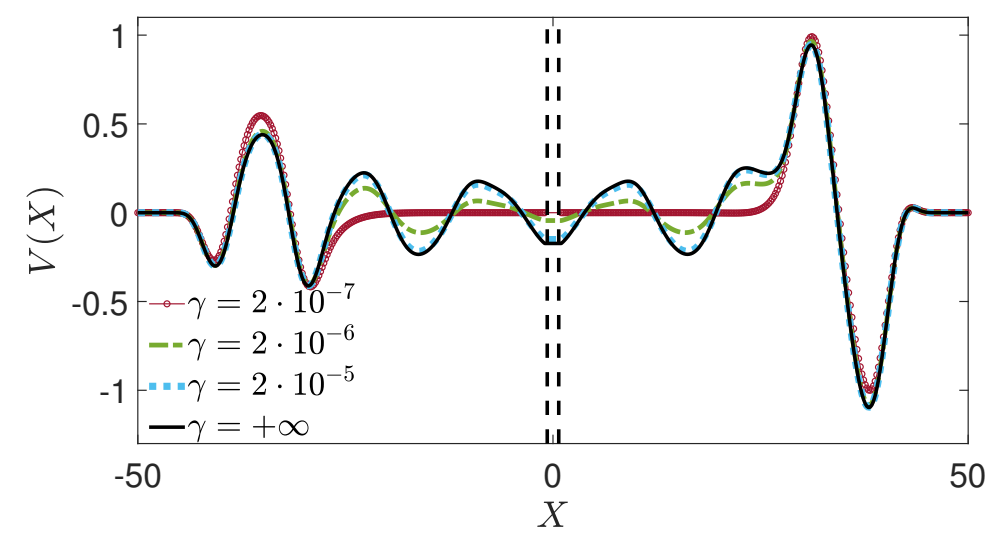

Figure 15: Velocity profiles of the homogenized problem at time $t=t_{f}$ for different values of $\gamma$.

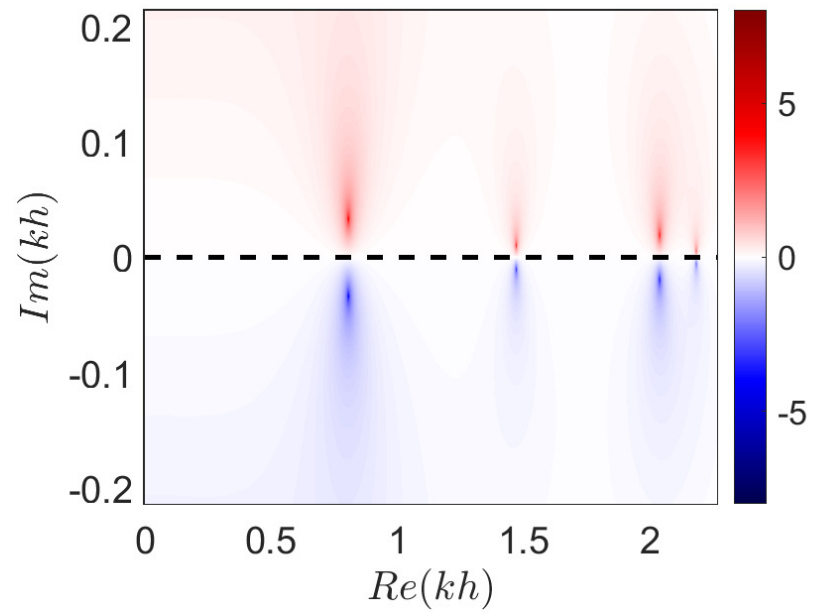

(a) $\gamma=+\infty$

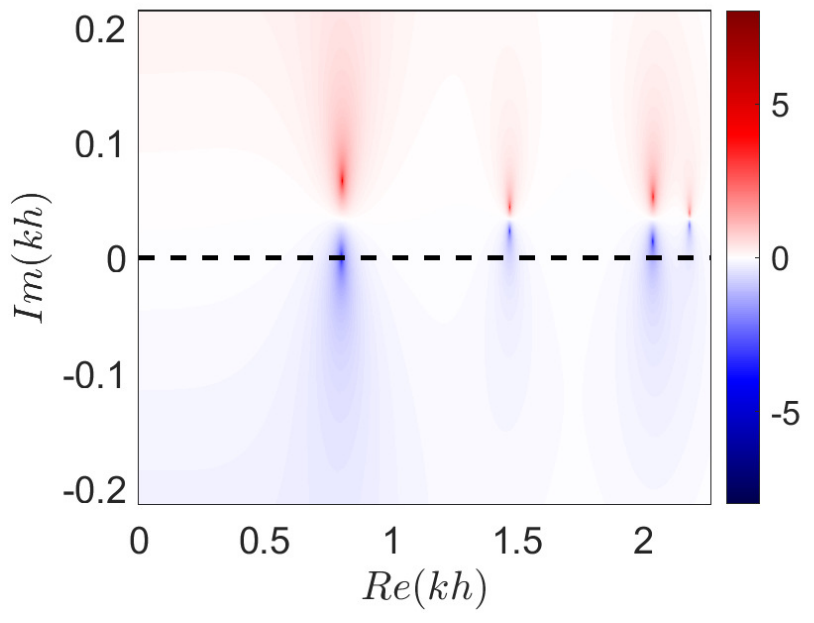

(b) $\gamma=4.47 \cdot 10^{-6}$

Figure 16: Complex frequency map of $\log (|\operatorname{det}(\mathbb{S})|)$ for the invisicid case (a) and for the critical value of the dissipation parameter associated with the first resonance(b), where $\mathbb{S}$ is the scattering matrix. We observe four poles and four zeros, in the vicinity of the first four resonance frequencies considered in $\mathscr{D}_{\gamma}(\omega)$.

To illustrate this behaviour around the resonant frequencies, we consider a causal monochromatic source point located at $X_{S}=-120 \mathrm{~m}$. In (51), the forcing $G\left(t c_{m}\right)$ is thus replaced by $A \sin \left(\omega_{S} t\right)$ if $t \geq 0$, and 0 if $t<0$. The angular frequency $\omega_{S}$ will be given for each case later on. The velocity profiles at $t=633 \mathrm{~ms}$ are displayed in Figure 19. The magnitude $A$ of the source is such that the amplitude of the emitted velocity is $0.5 \mathrm{~m} / \mathrm{s}$, which is denoted by horizontal dotted lines. At the considered instant, the incident wave to the left of the source has almost left the domain, and the front of the reflected wave is located near $-800 \mathrm{~m}$; it is marked by a green arrow. For $\gamma=+\infty$ (Figure 19a), the angular frequency is $\omega_{S}=\omega_{\text {min,1 }}$ for which minimal transmission is reached around the first resonance. The time-domain simulations confirm that there is almost no transmitted signal quite quickly. In Figure 19b, we consider the critical value of dissipation $\gamma=4.47 \cdot 10^{-6} \mathrm{~s} \cdot \mathrm{kg}^{-1} \cdot \mathrm{m}^{3}$ and the associated angular frequency $\omega_{S}=\tilde{\omega}_{1}$ for the source. The transmitted wave is greater, as expected. Between the front of the reflected wave (green arrow) and the interface, the amplitude of $V$ is greater than 0.5 , which confirms that the reflected wave is not absorbed. 


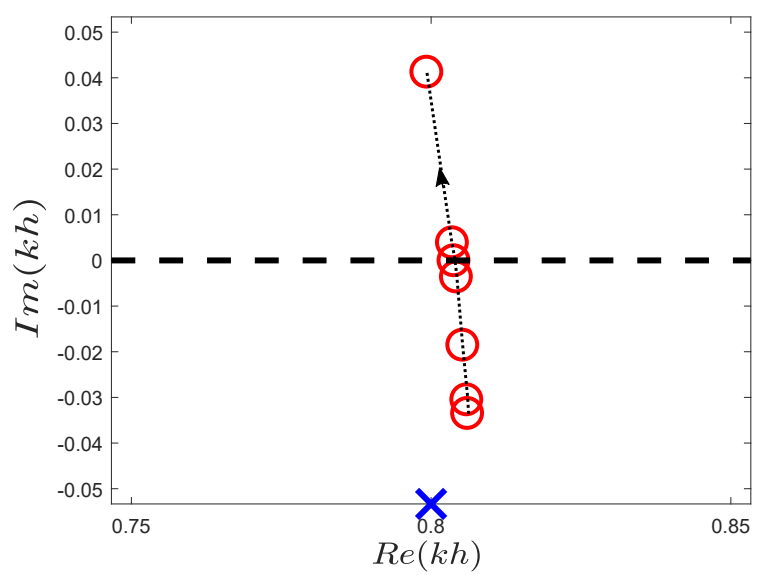

(a) first resonant frequency in $\mathscr{D}_{\gamma}(\omega)$

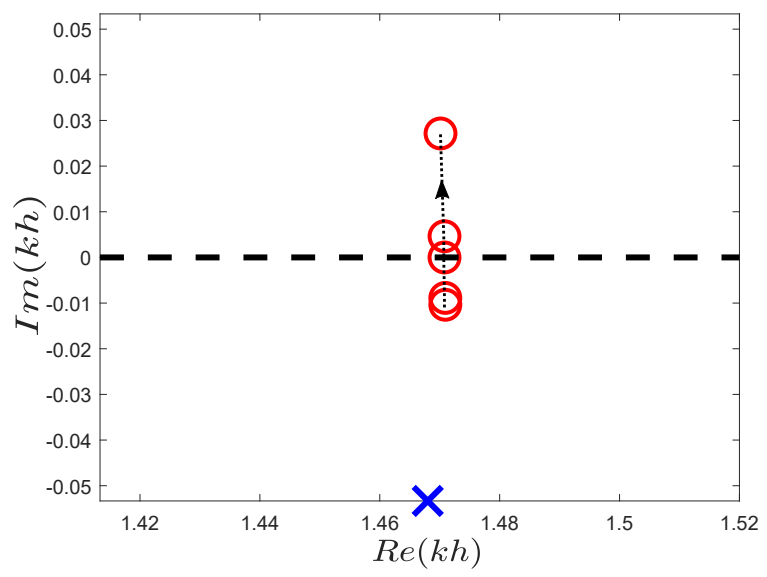

(b) second resonant frequency in $\mathscr{D}_{\gamma}(\omega)$

Figure 17: Zeros of $\operatorname{det}(\mathbb{S})$ in the complex plane, where $\mathbb{S}$ is the scattering matrix, for various values of the dissipation parameter. The arrow shows the direction of the trajectory as losses are increased. The red circles denote the zeros for $\gamma \in\left\{+\infty, 5 \cdot 10^{-5}, 1 \cdot 10^{-5}, 5 \cdot 10^{-6}, 4.47 \cdot 10^{-6}, 4 \cdot 10^{-6}, 2 \cdot 10^{-6}\right\}$ from bottom to top. The blue cross denotes the resonant frequency.

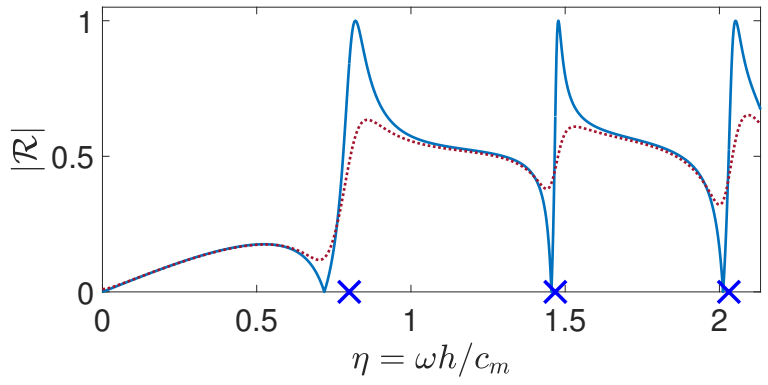

(a) Reflection coefficient

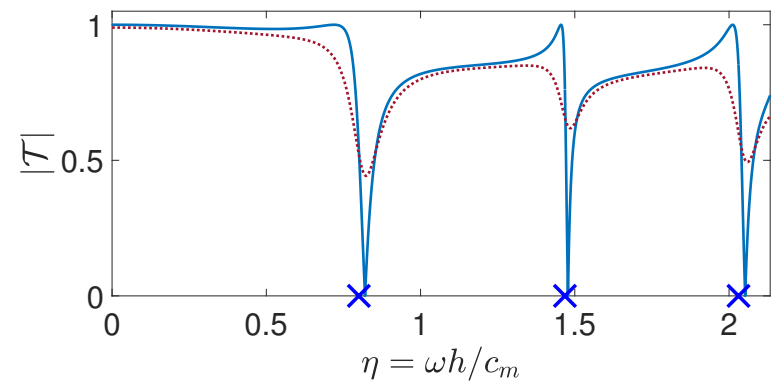

(b) Transmission coefficient

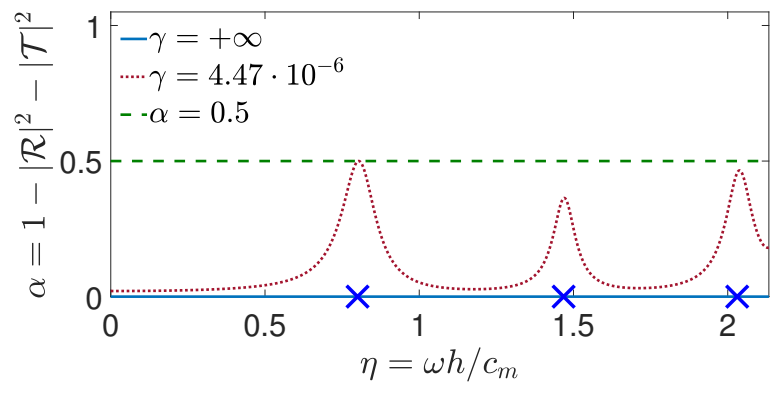

(c) Absorption coefficient

Figure 18: Reflection, transmission and absorption coefficients (25). The blue crosses denote $\omega_{r}$ in $\mathscr{D}_{\gamma}(\omega)$.

To obtain Coherent Perfect Absorption in our transmission problem [34], it is necessary to illuminate the resonant interface from both sides. To do this, two sources of angular frequency $\tilde{\omega}_{1}$ are placed symmetrically with respect to the interface and switched on at the same time. Figure 20 represents $V$ at $t=631 \mathrm{~ms}$. The dotted horizontal lines in \pm 0.5 mark the amplitude of the incident 


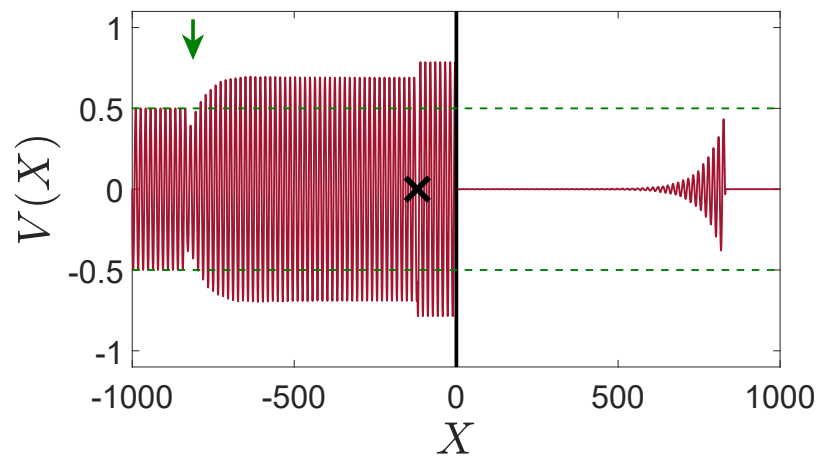

(a) $\gamma=+\infty$

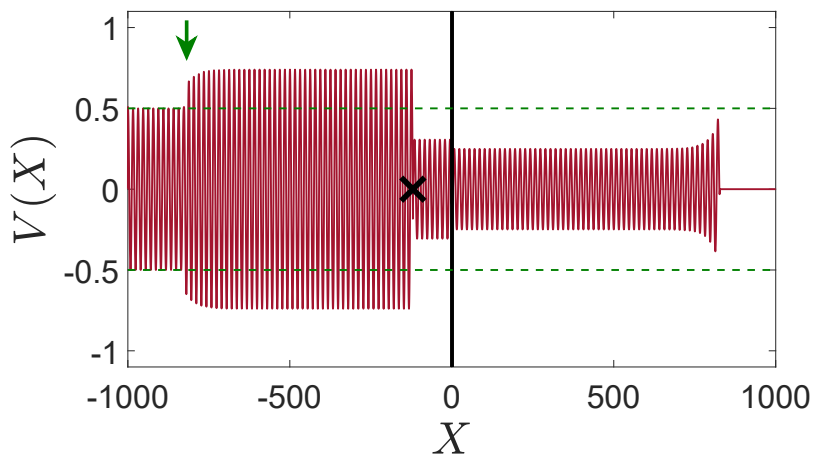

(b) $\gamma=4.47 \cdot 10^{-6} \mathrm{~s} \cdot \mathrm{kg}^{-1} \cdot \mathrm{m}^{3}$

Figure 19: Velocity profiles of the homogenized problem at $t=633 \mathrm{~ms}$ with one source. Dissipation parameter $\gamma=+\infty$ (a) and $\gamma=4.47 \cdot 10^{-6} \mathrm{~s} \cdot \mathrm{kg}^{-1} \cdot \mathrm{m}^{3}$ (b). The monochromatic source point is located at the black cross and is switched on at $t=0$. The angular frequency of the source $\omega_{S}$ is either $\omega_{\min , 1}$ (a) or $\tilde{\omega}_{1}$ (b). The green arrows denote the reflected wavefront.

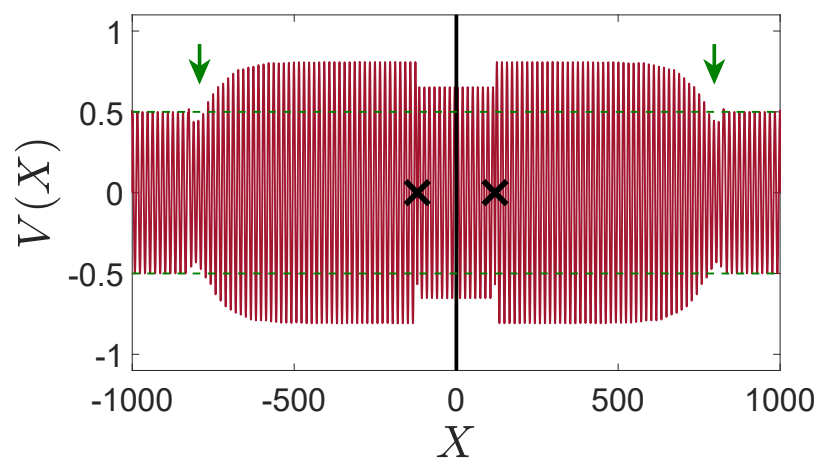

(a) $\gamma=+\infty$

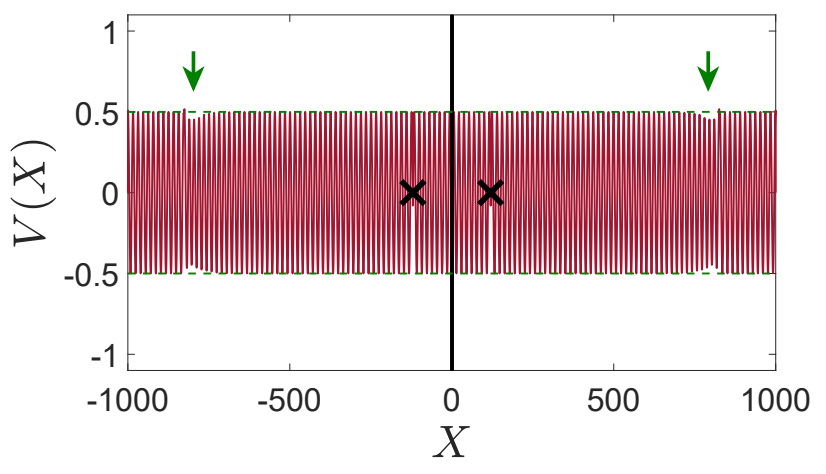

(b) $\gamma=4.47 \cdot 10^{-6} \mathrm{~s} \cdot \mathrm{kg}^{-1} \cdot \mathrm{m}^{3}$

Figure 20: Velocity profiles of the homogenized problem at $t=631 \mathrm{~ms}$ with two sources. Dissipation parameter $\gamma=+\infty$ (a) and $\gamma=4.47 \cdot 10^{-6} \mathrm{~s} \cdot \mathrm{kg}^{-1} \cdot \mathrm{m}^{3}$ (b). The two monochromatic source points are located at the black crosses and are switched on at $t=0$. The angular frequency of the source $\tilde{\omega}_{S}=\tilde{\omega}_{1}$ is such that $\operatorname{det}(\mathbb{S})\left(\tilde{\omega}_{S}\right)=0$ if $\gamma=4.47 \cdot 10^{-6} \mathrm{~s} \cdot \mathrm{kg}^{-1} \cdot \mathrm{m}^{3}$. The green arrows denote the reflected wavefronts.

wave emitted. At this moment, the incident waves emitted by the two sources have almost left the domain. On the other hand, the fronts of the reflected waves are visible and marked by green arrows. In the unattenuated case (Figure 20a), the reflected waves are observed, which lead to an increase in the amplitude of the waves. In the dissipative case with critical coupling (Figure 20b), the wave amplitude does not exceed 0.5. This means that the reflected waves have disappeared and have been completely absorbed by the dissipative resonant interface.

\section{Conclusion}

We considered a periodic row of inclusions with a scaling in the shear moduli as $\eta^{2}$, where $\eta$ is the usual small parameter. Compared with existing results, dissipation of energy was considered. The effective jump conditions applying have thus been enriched in the frequency-domain and the timedomain using the homogenization techniques. A time-domain numerical method has been proposed in 
1D and 2D to deal with the model so obtained. Finally, time-domain simulations have been presented to validate the numerical method. Once validated, this method has then been used to compare the solution with simulations in the original microstructure. A good agreement has been found and discussed. Simulations of the homogenized problem have also been performed to investigate the role played by the dissipation parameter. An application to Coherent Perfect Absorption has been proposed, in lines with current researches done on subwavelength metasurfaces [34].

As in the case where no dissipation is considered, perspectives include the derivation of an effective model at higher order, as discussed in [10, 31, 41]. This could allow to account for the resonances associated with modes with zero mean, which are missed in the current model. However, it seems that when dissipation is considered, this does not induce a large error compared with full-field solutions. Another fruitful direction of work concerns the design of meta-interfaces to reach some objective effective properties through a topological optimization process. This approach has been developped in [6] for bulk metamaterials and its adaptation for thin resonant metasurfaces could be of particular interest, for example, in the noise reduction field [25, 37].

\section{Acknowledgment}

We acknowledge the École Centrale Marseille for the funding of the internship of X. Gao. We thank Cédric Bellis for fruitful discussions. Lastly, Antonin Coutant is greatly acknowledged for his insights about Coherent Perfect Absorption.

\section{Appendix A. Numerical approximation of the phantom values in 1D}

This section details the computation of the phantom values defined in (38). It requires to derive the $q$-th order jump conditions. The jump conditions in (34) are differentiated iteratively $q$ times with respect to $t$. At each step, the temporal derivatives are replaced by the spatial derivatives using (34). This process can be written:

$$
\mathbb{C}_{+}^{q} \boldsymbol{U}_{+}^{q}\left(t_{n}\right)+\boldsymbol{R}_{+}^{q}\left(t_{n}\right)=\mathbb{C}_{-}^{q} \boldsymbol{U}_{-}^{q}\left(t_{n}\right)+\boldsymbol{R}_{-}^{q}\left(t_{n}\right)+\mathbb{Q}^{q} \boldsymbol{Z}\left(t_{n}\right)
$$

with $\mathbb{C}_{ \pm}^{q}$ being $q_{U} \times q_{U}$ matrices and $\mathbb{C}_{+}^{q}$ being invertible in the cases considered hereafter, $\mathbb{Q}^{q}$ being a $q_{U} \times 2 N_{R}$ matrix, and $\boldsymbol{R}_{ \pm}^{q}\left(t_{n}\right)$ being $q_{U}$-element vectors containing the $(q+1)$-th spatial derivatives of the fields. The vector $\boldsymbol{Z}$ contains the $2 N_{R}$ auxiliary variables at time $t_{n}$, i.e.

$$
\boldsymbol{Z}\left(t_{n}\right)=\left(J_{1}\left(t_{n}\right), \ldots, J_{N_{R}}\left(t_{n}\right), G_{1}\left(t_{n}\right), \ldots, G_{N_{R}}\left(t_{n}\right)\right)^{\top} .
$$


The expression of the matrices and vectors which depend on the physical parameters are given below in the case $q=3$ :

$$
\begin{aligned}
& \left\{\begin{array}{lll}
\mathbb{C}_{ \pm}^{3}[i, i]=1, & \mathbb{C}_{ \pm}^{3}[5,1]=\mp \frac{\widetilde{D}_{0}}{2}, \\
\mathbb{C}_{ \pm}^{3}[1,2]=\mp \frac{\widetilde{B}_{1}}{2}, & \mathbb{C}_{ \pm}^{3}[5,6]=\mp \frac{\widetilde{C}_{11}+h \alpha_{0}}{2}, \\
\mathbb{C}_{ \pm}^{3}[2,3]=\mp \frac{\widetilde{C}_{11}+h \alpha_{0}}{2}, & \mathbb{C}_{ \pm}^{3}[6,7]=\mp \frac{\widetilde{B}_{1}}{2}, \\
\mathbb{C}_{ \pm}^{3}[2,6]=\mp \frac{\widetilde{D}_{0}}{2 \rho_{m} \mu_{m}}, & \mathbb{C}_{ \pm}^{3}[7,3]=\mp \frac{h \rho_{m}}{\gamma \rho_{i}}\left(\frac{\alpha_{0}}{2}-\sum_{r=1}^{N_{R}} \alpha_{r}^{2}\right), \\
\mathbb{C}_{ \pm}^{3}[3,4]=\mp \frac{\widetilde{B}_{1}}{2}, & \mathbb{C}_{ \pm}^{3}[7,6]= \pm \frac{h}{2\left(\gamma \rho_{i} c_{m}\right)^{2}} \sum_{r=1}^{N_{R}} \alpha_{r}^{2}, \\
\mathbb{C}_{ \pm}^{3}[4,3]=\mp \frac{h}{2\left(\gamma \rho_{i} c_{m}\right)^{2}} \sum_{r=1}^{N_{R}} \alpha_{r}^{2}, & \mathbb{C}_{ \pm}^{3}[7,8]=\mp \frac{1}{2}\left(\widetilde{C}_{11}+h \alpha_{0}-h \sum_{r=1}^{N_{R}} \alpha_{r}^{2}\right), \\
\mathbb{C}_{ \pm}^{3}[4,6]=\mp \frac{h}{2 \mu_{m} c_{m}^{2}\left(\gamma \rho_{i}\right)^{3}} \sum_{r=1}^{N_{R}} \alpha_{r}^{2}, & \mathbb{C}_{ \pm}^{3}[i, j]=0 \text { else. } \\
\mathbb{C}_{ \pm}^{3}[4,8]=\mp \frac{h}{2 \mu_{m} \gamma \rho_{i}}\left(\alpha_{0}-\sum_{r=1}^{N_{R}} \alpha_{r}^{2}\right), &
\end{array}\right. \\
& \begin{cases}\mathbb{Q}^{3}[2, j]=-\frac{h}{\mu_{m}} & \text { if } j \in\left\{N_{R}+1, \ldots, 2 N_{R}\right\}, \\
\mathbb{Q}^{3}[4, j]=-\frac{h \omega_{j}^{2}}{\mu_{m} c_{m}^{2} \gamma \rho_{i}} & \text { if } j \in\left\{1, \ldots, N_{R}\right\}, \\
\mathbb{Q}^{3}[4, j]=\frac{h}{\mu_{m} c_{m}^{2}}\left(\omega_{j-N_{R}}^{2}-\frac{1}{\left(\gamma \rho_{i}\right)^{2}}\right) & \text { if } j \in\left\{N_{R}+1, \ldots, 2 N_{R}\right\}, \\
\mathbb{Q}^{3}[5, j]=-h & \text { if } j \in\left\{1, \ldots, N_{R}\right\}, \\
\mathbb{Q}^{3}[7, j]=\frac{h \omega_{j}^{2}}{c_{m}^{2}} & \text { if } j \in\left\{1, \ldots, N_{R}\right\}, \\
\mathbb{Q}^{3}[7, j]=\frac{h}{c_{m}^{2} \gamma \rho_{i}} & \text { if } j \in\left\{N_{R}+1, \ldots, 2 N_{R}\right\}, \\
\mathbb{Q}^{3}[i, j]=0 & \text { else. } \\
\mp \frac{1}{2}\left(\widetilde{C}_{11}+h \alpha_{0}-h \sum_{r=1}^{N_{R}} \alpha_{r}^{2}\right) \partial_{X}^{4} V_{ \pm}, & \boldsymbol{R}_{ \pm}^{3}[8]=\mp \frac{\widetilde{B}_{1}}{2} \partial_{X}^{4} \Sigma_{ \pm}, \quad \boldsymbol{R}_{ \pm}^{3}[i]=0 \text { else. }\end{cases}
\end{aligned}
$$

One recalls that index numbering starts at 1 for all the vectors and matrices considered in this paper. When $\gamma=+\infty$, one recovers the equations (19), (20) and (21) in [40].

To compute the phantom values (38), Taylor expansions are written at $q_{T}$ nodes $X_{I_{L}-q_{T}+1}, \ldots, X_{I_{L}}$ on the left side of the enlarged interface and at $q_{T}$ nodes $X_{I_{R}}, \ldots, X_{I_{R}+q_{T}-1}$ on the right side of the enlarged interface. The phantom values $\boldsymbol{u}_{*}\left(X_{j}, t_{n}\right)$ are then computed using these Taylor expansions 
and the $q$-th order jump conditions (A.1). We introduce the following matrices and vectors by blocks:

$$
\begin{aligned}
& \mathbb{M}^{q}=\left(\begin{array}{c}
\mathbb{T}_{I_{L}-q_{T}+1}^{q}\left(-\frac{a}{2}\right) \\
\vdots \\
\mathbb{T}_{I_{L}}^{q}\left(-\frac{a}{2}\right) \\
\mathbb{T}_{I_{R}}^{q}\left(\frac{a}{2}\right)\left(\mathbb{C}_{+}^{q}\right)^{-1} \mathbb{C}_{-}^{q} \\
\vdots \\
\mathbb{T}_{I_{R}+q_{T}-1}^{q}\left(\frac{a}{2}\right)\left(\mathbb{C}_{+}^{q}\right)^{-1} \mathbb{C}_{-}^{q}
\end{array}\right), \quad \mathbb{F}^{q}=\left(\begin{array}{c}
\mathbf{0} \\
\vdots \\
\mathbf{0} \\
-\mathbb{T}_{I_{R}\left(\frac{a}{2}\right)}^{q} \\
\vdots \\
-\mathbb{T}_{I_{R}+q_{T}-1}^{q}\left(\frac{a}{2}\right)
\end{array}\right)\left(\mathbb{C}_{+}^{q}\right)^{-1}, \\
& \mathbb{G}^{q}=\mathbb{C}_{-}^{q}\left(\mathbb{M}^{q}\right)^{-1} \mathbb{F}^{q}+\mathbb{I}_{q_{U}} \quad \text { and } \quad \mathcal{U}^{n}=\left(\begin{array}{c}
(\boldsymbol{u})_{I_{L}-q_{T}+1}^{n} \\
\vdots \\
(\boldsymbol{u})_{I_{L}}^{n} \\
(\boldsymbol{u})_{I_{R}}^{n} \\
\vdots \\
(\boldsymbol{u})_{I_{R}+q_{T}-1}^{n}
\end{array}\right) .
\end{aligned}
$$

The matrices $\mathbb{M}^{q}$ and $\mathbb{F}^{q}$ are $4 q_{T} \times q_{U}$ matrices, $\mathbb{G}^{q}$ is a $q_{U} \times q_{U}$ matrix, $\mathcal{U}^{n}$ is a $4 q_{T}$-element vector and $\boldsymbol{Z}^{n}$ is the numerical approximation of $\boldsymbol{Z}\left(t_{n}\right)$. When $q_{T}$ is such that $4 q_{T}=q_{U}$, then $\mathbb{M}^{q}$ is a square matrix that is formally checked to be invertible. If $4 q_{T}>q_{U}$ then $\mathbb{M}^{q}$ is not square and $\left(\mathbb{M}^{q}\right)^{-1}$ denotes its Moore-Penrose pseudo-inverse. The numerical approximations $\left(\boldsymbol{u}_{*}\right)_{j}^{n}$ of the phantom values (39) are finally obtained neglecting the derivatives of order greater than $(q+1)$.

[1] Auriault, J.-L., Bonnet, G., 1985. Dynamique des composites élastiques périodiques. Arch Mech. 37 (4-5), 269-284.

[2] Auriault, J.-L., Boutin, C., 2012. Long wavelength inner-resonance cut-off frequencies in elastic composite materials. International Journal of Solids and Structures 49 (23-24), 3269-3281.

[3] Bensoussan, A., Lions, J.-L., Papanicolaou, G., 2011. Asymptotic analysis for periodic structures. AMS Chelsea Publishing.

[4] Carcione, J. M. (Ed.), 2007. Wave Fields in Real Media - Wave Propagation in Anisotropic, Anelastic, Porous and Electromagnetic Media. Elsevier.

[5] Chen, Y., Barnhart, M., Chen, J., Hu, G., Sun, C., Huang, G., 2016. Dissipative elastic metamaterials for broadband wave mitigation at subwavelength scale. Composite Structures 136, $358-371$.

[6] Cornaggia, R., Bellis, C., 2020. Tuning effective dynamical properties of periodic media by FFT-accelerated topological optimization. International Journal for Numerical Methods in Engineering 121 (14), 3178-3205.

[7] Craster, R. V., Guenneau, S. (Eds.), 2013. Acoustic Metamaterials. Springer Netherlands.

[8] David, M., Pideri, C., Marigo, J.-J., 2012. Homogenized interface model describing inhomogeneities located on a surface. Journal of Elasticity 109 (2), 153-187. 
[9] Delourme, B., 2010. Modèles asymptotiques des interfaces fines et périodiques en électromagnétisme. PhD thesis - Université Pierre et Marie Curie - Paris VI.

[10] Felbacq, D., Bouchitté, G., 2005. Theory of mesoscopic magnetism in photonic crystals. Physical Review Letters 94 (18).

[11] Huang, S., Fang, X., Wang, X., Assouar, B., Cheng, Q., Li, Y., 2019. Acoustic perfect absorbers via helmholtz resonators with embedded apertures. Journal of the Acoustical Society of America 145 (1), 254-262.

[12] Hussein, M. I., Frazier, M. J., 2012. Damped phononic crystals and acoustic metamaterials. In: Acoustic Metamaterials and Phononic Crystals. Springer Berlin Heidelberg, pp. 201-215.

[13] Jiang, X., Li, Y., Zhang, L., 2017. Thermoviscous effects on sound transmision through a metasurface of hybrid resonances. Journal of the Acoustical Society of America 141 (4), 363368.

[14] Jimenez, N., Huang, W., Romero-Garcia, V., Pagneux, V., Groby, J., 2016. Ultra-thin metamaterial for perfect and quasi-omnidirectional sound absorption. Applied Physics Letters 109, 121902.

[15] Jimenez, N., Romero-Garcia, V., Pagneux, V., Groby, J., 2017. Rainbow-trapping absorbers: broadband, perfect and asymmetric sound absorption by subwavelength panels for transmission problems. Scientific Reports 7, 13595.

[16] Krushynska, A., Kouznetsova, V., Geers, M., 2016. Visco-elastic effects on wave dispersion in three-phase acoustic metamaterials. Journal of the Mechanics and Physics of Solids 96, 29-47.

[17] Leng, J., Gautier, F., Pelat, A., Pico, R., Groby, J., Romero-Garcia, V., 2019. Limits of flexural wave absorption by open lossy resonators: reflection and transmission problems. New Journal of Physics 21, 053003.

[18] LeVeque, R. J., 2002. Finite Volume Methods for Hyperbolic Problems (Cambridge Texts in Applied Mathematics). Cambridge University Press.

[19] Lewinska, M., Kouznetsova, V., van Dommelen, J., Krushynska, A., 2017. The attenuation performance of locally resonant acoustic metamaterials based on generalised viscoelastic modeling. International Journal of Solids and Structures 126-127, 163-174.

[20] Li, Y., Shen, C., Xie, Y., Li, J., Wang, W., Cummer, S. A., Jing, Y., 2017. Tunable asymmetric transmission via lossy acoustic metasurfaces. Physical Review Letters 119 (3).

[21] Lombard, B., Maurel, A., Marigo, J.-J., 2017. Numerical modeling of the acoustic wave propagation across an homogenized rigid microstructure in the time domain. Journal of Computational Physics 335, 558-577.

[22] Lombard, B., Piraux, J., 2004. Numerical treatment of two-dimensional interfaces for acoustic and elastic waves. Journal of Computational Physics 195 (1), 90-116. 
[23] Lombard, B., Piraux, J., Gélis, C., Virieux, J., 2008. Free and smooth boundaries in 2-D finite-difference schemes for transient elastic waves. Geophysical Journal International 172 (1), 252-261.

[24] Lorcher, F., Munz, C.-D., 2006. Lax-wendroff-type schemes of arbitrary order in several space dimensions. IMA Journal of Numerical Analysis 27 (3), 593-615.

[25] Ma, G., Yang, M., Xiao, S., Yang, Z., Sheng, P., 2014. Acoustic metasurface with hybrid resonances. Nature Materials 13 (9), 873-878.

[26] Marigo, J.-J., Maurel, A., 2016. Homogenization models for thin rigid structured surfaces and films. The Journal of the Acoustical Society of America 140 (1), 260-273.

[27] Marigo, J.-J., Maurel, A., Pham, K., Sbitti, A., 2017. Effective dynamic properites of a row of elastic inclusions: The case of scalar shear waves. Journal of Elasticity 128 (2), 265-289.

[28] Marigo, J.-J., Pideri, C., 2011. The effective behaviour of elastic bodies containing microcracks or microholes localized on a surface. International Journal of Damage Mechanics, SAGE Publications 20, 1151-1177.

[29] Maurel, A., Mercier, J.-F., Pham, K., Marigo, J.-J., Ourir, A., 2019. Enhanced resonance of sparse arrays of helmholtz resonators-application to perfect absorption. Journal of the Acoustical Society of America 145 (4), 2552-2560.

[30] Moleron, M., Serra-Garcia, M., Daraio, C., 2016. Visco-thermal effects in acoustic metamaterials: from total transmision to total reflection and high absorption. New Journal of Physics 18, 033003.

[31] Pham, K., Maurel, A., Marigo, J.-J., 2017. Two scale homogenization of a row of locally resonant inclusions - the case of shear waves. Journal of the Mechanics and Physics of Solids 106, 80-94.

[32] Press, W. H., Teukolsky, S. A., Vetterling, W. T., Flannery, B. P., 2007. Numerical Recipes. Cambridge University Pr.

[33] Romero-Garcia, V., Jimenez, N., Groby, J., Merkel, A., Tournat, V., Theocharis, G., Richoux, O., Pagneux, V., 2020. Perfect absorption in mirror-symmetric acoustic metascreens. Physical Review Applied 14, 054055.

[34] Romero-Garcia, V., Jimenez, N., Theocharis, G., Achilleos, V., Merkel, A., Richoux, O., Tournat, V., Groby, J., Pagneux, V., 2020. Design of acoustic metamaterials made of helmholtz resonators for perfect absorption by using complex frequency plane. Comptes Rendus Physique 21 (7-8), 713-749.

[35] Romero-Garcia, V., Theocharis, G., Richoux, O., Merkel, A., Tournat, V., Pagneux, V., 2016. Perfect and broadband absorption by critically coupled sub-wavelength resonators. Scientific Reports 6, 19519.

[36] Sanchez-Hubert, J., Sanchez-Palencia, E., 1992. Introduction aux méthodes asymptotiques et à l'homogénéisation. Collection Mathématiques Appliquées pour la Maîtrise. 
[37] Schwan, L., Umnova, O., Boutin, C., 2017. Sound absorption and reflection from a resonant metasurface: Homogenisation model with experimental validation. Wave Motion 72, 154-172.

[38] Schwartzkopff, T., Dumbser, M., Munz, C.-D., 2004. Fast high order ADER schemes for linear hyperbolic equations. Journal of Computational Physics 197 (2), 532-539.

[39] Su, X., Lu, Z., Norris, A. N., 2018. Elastic metasurfaces for splitting SV- and p-waves in elastic solids. Journal of Applied Physics 123 (9), 091701.

[40] Touboul, M., Lombard, B., Bellis, C., 2020. Time-domain simulation of wave propagation across resonant meta-interfaces. Journal of Computational Physics 414, 109474.

[41] Touboul, M., Pham, K., Maurel, A., Marigo, J.-J., Lombard, B., Bellis, C., 2020. Effective resonant model and simulations in the time-domain of wave scattering from a periodic row of highly-contrasted inclusions. Journal of Elasticity 142 (1), 53-82. 